\title{
Anomalies in Quantum Field Theory and Differential Geometry •
}

\author{
Juan L. Mañes \\ Ph.D. Thesis \\ Lawrence Berkeley Laboratory \\ and \\ Department of Physics \\ University of California \\ Berkeley, California 9\$720
}

\begin{abstract}
DISCLAIMER
This report was prepared as an account of work sponsored by an agency of the United States Government. Neither the United States Government nor any agency thereof, nor any of their employees, makes any warranty, express or implied, or assumes any legal liability or responsibility for the accuracy, completeness, or urefulness of any information, apparatus, product, or process disclosed, or represents that its use would not infringe privately owned rights. Reference herein to any specific commercial product, process, or service by trade name, trademark, manufacturer, or otherwise does not necessarily constitute or imply its endorsement, recommendation, or favnring by the United States Government or any agency thereof. The views and opinions of authors expressed hercin do not necessarily state or reflect those of the United States Government or any agency thereof.
\end{abstract}

\footnotetext{
- This work was supported by the Director, Office of Energy Research, Office of High Energy and Nuclear Physics, Division of High Energy Physics of the U.S. Department of Energy under Contract DE-AC03765 F00098.

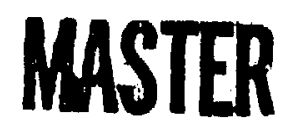




\section{ANOMALIES IN QUANTUM FIELD THEORY AND \\ DIFFERENTIAL GEOMETRY}

by

Juan Luis Mañes

\section{ABSTRACT}

Anomalies in field theory appeared first in perturbative computations involving Feynman diagrams. It is only recently that differential geometric techniques have been used to obtain the form of gauge and gravitational anomalies in a direct and simple way. This is possible because of the topological nature of the anomaly.

In the first chapter of this thesis the gauged Wess-Zumino action is constructed by differentiai geometry methods. After reviewing the relevant techniques, an expression for the action valid in any (oven) number of space-time dimensions is obtained. This expression is compired with Witten's result in four dimensions.

The link between topology and the anomaly is provided by the appropriate index theorem. The index density is a supersymmetric invariant polynomial from which the anomaly and other related objects can be obtained 
through the use of the "descent equations". A new proof of the Atiyah-Singer index theorem for the Dirac operator is presented in chapter 3. This proof is based on the use of a WKB approximation to evaluate the supertrace of the kernel for a supersymmetric hamiltonian. Chapter 2, which is dedicated to the development of the necessary WKB techniques, contains also a discussion of mechanical systems with bosonic and fermionic degrees op freedom. 
Dedicated to my parents, Luis and Pilar 


\section{Acknowledgements}

I would like to thank my advisor, Prof. Bruno Zumino, for his continuous support and understanding throughout my graduate study, and for many long and useful conversations. Profs. Orlando Alvarez, Korkut Bardakci and Mary Gaillard have greatly contributed to my education as a physicist through courses and discussions.

I have done my research at Lawrence Berkeley Laboratory during the past two and a half years. My thanks go to all who have made it a stimulating environment for physics. In particular, I want to thank Randy Ingermanson and Augusto Sagnotti, who in different occasions have given me help and advice. Also the secretaries have been friends as well as a great help -thanks are expressed to Mary Gorman, Kathy Hardy, Betty Moura and Luanne Newmann.

I owe personal thanks to Shahram Hamidi, Milan Mijic and Hidenori Sonoda, who from the beginning of my studies in this country have been excellent friends.

Finally, I would like to express my deep gratitude to Prof. Pablo Barrón. His continuous encouragement and stimulus during my first years as an engineering student helped me discover my inclination for theoretical physics. 
Contents

Dedication

Acknowledgements

Introduction

1 Differential geometry and the Wess-Zumino action

1.J. Differential geometry and the effective functional $W\left(R, L, g_{R}, g_{L}\right) \quad 6$

1.2 The functional $W(R, L, U) \ldots \ldots \ldots$

1.3 The counterterm ................... 14

1.4 An application ....................... 16

2 WKB method for Bose-Fermi quantum-mechanical systems 22

2.1 Classical mechanics with fermionic variables . . . . . . . 23

2.2 WKB expression for the kernel . . . . . . . . . . 27

2.3 WKB calculation of traces . . . . . . . . . . . 35

3 WKB proof of the index theorem 41

3.1 WKB formula for the index . . . . . . . . . . . 43

3.2 Validity of the WKB approximation . . . . . . . . 48

3.3 Computation of the index ............. 51

$\begin{array}{ll}\text { Appendix A } & 58\end{array}$

$\begin{array}{ll}\text { Appendix B } & 60\end{array}$ 
Appendix D

Appendix E

References

74 


\section{INTRODUCTION}

Chiral anomalies in field theory appeared first in perturbative computations involving Feynman diagrams (see $[1,2]$ and references therein). Later, Wess and Zumino [3] realized that non-abelian chiral anomalies had to satisfy certain consistency conditions. These consistency conditions could be used to determine the non-abelian anomaly once the abelian case was known. They also provided a way of checking the many different expressions obtained pertusbatively.

However, it is only recently $[4,5,6]$ that differential geometric techniques have been used to obtain solutions to the consistency conditions in an elegant and simple way. This has provided an alternative method to the lengthy and complicated Feynman-diagrammatic computations. These techniques have also been extended to the study of gravitational anomalies $[7,8]$ and anomalies in supersymmetric gauge theories [9].

In a different line of developments, Witten [10] has obtained an expression for the Wess-Zumino effective action from topological considerations. This effective action was originally constructed [3] from the non-abelian anomaly by using the Wess-Zumino consistency conditions. Witten's result "vas surprising not only because of the topology involved, but also because the action appeared as a finite polynomial in the gauge fields.

In chapter 1 of this thesis I extend the differential geometric techniques to the calculation of the Wess-Zumino action. A general expression valid in any (even) number of space-time dimensions is obtained. This expression 
coincides with Witten's result in four dimensions and it is simpler to evaluate: in that it does not require the use of a trial and error method A review of the relevant differential geometric techniques is also presented in chapter 1.

The link between topology and the anomaly is provided by the appropriate index theorem $(11,12)$. The index density is a symmetric invariant polynomial from which the anomaly and other related objects can be obtained through the use of the "descent equations" $[5,6]$.

The use of supersymmetry has made it possible to give simple proofs of the index theorem [13-15], based on concepts familiar to physicists. However, these proofs are somewhat obscured by the presence of path integrals, which are always tricky to define and hard to evaluate in a rigorous way.

A conceptually simpler approach can be found in ref. [16], which presents a proof of the Atiyah-Singer index theorem for the Dirac operator. This proof uses a WIKB approximation instead of path-integrals, but only the case of vanishing external gauge felds is treated. The generalization to nonvanishing gauge fields is no trivial, and is the subject of chapter 3 of this thesis. The necessary WKB techniques are developed in chapter 2, which contains a study of mechanical systems with bosonic and formionic degrees of freedora. 


\section{DIFFERENTIAL GEOMETRY AND THE WESS-ZUMINO ACTION}

Some new developments have taken place recently concerning the mathematical structure of chiral anomalies. The use of differential geometry methods has made passible the explicit calculation of both abelian and non-abelian chiral anomalies without evaluating Feynman diagrams $[4,5]$, and the form of the Wess-Zumino [3] terms as well as its new a priori quantization has been derived by Witten $[10$ ) on the basis of topological considerations. Furthermore, Witten has used a trial and error method to gauge the Wess-Zumino term, and has found that the dependence of the resulting action on the gauge Gelds is in the form of a finite polynomial.

In this chapter, a general expression for the Wess-Zumino action is obtained by differential geometry techniques. This expression, which is evaluated directly without the use of a trial and error method, yields the action in the form given by Witten [10], except for some minor discrepancies that we discuss in detail.

We consider a theory of fermions interacting with external gauge fields. The spinor feld has internal degrees of freedom and transforms under a representation of a (flavor) group $G$ with generators $\left\{\lambda_{k}\right\}$. The lagrangian includes vector and axial vector gauge fields $V_{\mu}^{k}$ and $A_{\mu}^{k}$ :

$$
\mathcal{L}=i \bar{\psi} \gamma^{\mu}\left(\partial_{\mu}-i \lambda_{k} V_{\mu}^{k}-i \gamma_{s} \lambda_{k} A_{\mu}^{k}\right) \psi
$$

We will use the symbol $\gamma_{5}$ for the equivalent matrix in $2 n$-dimensional space- 
time, i.e.

$$
\gamma_{5} \equiv-i^{n+1} \prod_{\mu=0}^{3 n-1} \gamma^{\mu}, \text { and } \gamma_{5}^{2}=1
$$

The fields $V_{\mu}^{k}$ and $A_{\mu}^{k}$ will be combined in the Lie algebra-valued 1-forms $V$, $A$ and $V_{+}$:

$$
\begin{aligned}
V & \equiv-i \lambda_{k} V_{\mu}^{k} d x^{\mu} \equiv V_{\mu} d x^{\mu} \equiv-i \lambda_{k} V^{k} \\
A & \equiv-i \lambda_{k} A_{\mu}^{k} d x^{\mu} \equiv A_{\mu} d x^{\mu} \equiv-i \lambda_{k} A^{k} \\
V_{+} & \equiv V+\gamma_{s} A
\end{aligned}
$$

A general local transformation on the spinor fields is written

$$
\psi \rightarrow \exp \left(i \beta^{k} \lambda_{k}+i \alpha^{k} \lambda_{k} \gamma_{B}\right) \psi
$$

and if we define the 0-forms

$$
\beta \equiv-i \beta^{k} \lambda_{k}, \quad \alpha \equiv-i \alpha^{k} \lambda_{k}, \quad v \equiv \beta+\alpha \gamma_{B} \equiv-i v^{k} \lambda_{k}
$$

the transformation of the gauge fields can be written in a compact way:

$$
V_{+} \rightarrow g^{-1} V_{+} g+g^{-1} d g
$$

where $g=e^{\nu} \in G \times G$.

It is well known that the vector and axial vector currents

$$
J_{\mu k}^{v}=\bar{\psi} \gamma_{\mu} \lambda_{k} \psi \quad, \quad J_{\mu k}^{A}=\bar{\psi} \gamma_{\mu} \gamma_{5} \lambda_{k} \psi
$$

satisfy anomalous non-conservation laws:

$$
D^{\mu} J_{\mu k}^{V}=-G_{k}^{V}(V, A) \quad, \quad D^{\mu} J_{\mu k}^{A}=-G_{k}^{A}(V, A)
$$


The functions $G_{k}^{V}$ and $G_{k}^{A}$ were obtained by Gross and Jackiw [2] and Bardeen [1], who was able to remove the anomaly from the vector current by adding a suitable counterterm to the lagrangian.

Wess and Zumino [3| showed that the functions $G_{k}^{V, A}$ could be obtained from a functional $W(A, V, \xi)$ containing the gauge fields and an additional - field $\xi$ (a meson octet in the case $G \in S U(3)$ ), such that $e^{i \xi} \in G$, as

$$
G_{k}^{V}=\left.\frac{\delta W\left(V_{g}, A_{g}, \xi_{g}\right)}{\delta \beta^{k}}\right|_{\alpha=\rho=0} \quad, \quad G_{k}^{A}=\left.\frac{\delta W\left(V_{g}, A_{g}, \xi_{g}\right)}{\delta \alpha^{k}}\right|_{\alpha=\rho=0}
$$

where $V_{g}, A_{g}, \xi_{g}$ are the transformed of $V, A, \xi$ by the local action of $g \in G \times G$. In their original form the Wess-Zumino effective action $W(V, A, \xi)$ was not apparently a finite polynomial in the gauge fields. As it was already noted, this fact only became clear with the explicit evaluation of the action by Witten [10], who obtained it as a functional $W(R, L, U)$ depending on the left and right gauge fields and $U=e^{i \epsilon}$.

More recently, it has been possible to obtain the finctions $G_{k}^{V, A}$ by differential geometry methods $[4,5]$ based on the use of the Wess-Zumino consistency conditions that these functions must satisfy. Also, Zumino [5] has given an explicit expression for the action in the form $W\left(R, L, g_{R}, g_{L}\right)=$ $W\left(R, g_{R}\right)-W\left(L, g_{L}\right)$, where $g_{L}$ and $g_{R}$ are elements of $\mathrm{G}$. This expression is obtained directly as a polynomial in the gauge fields, but differs from the one given by Witten in that here we need two octets $\xi_{R}$ and $\xi_{L}$ of meson fields.

Since these differential geometry techniques are relatively unknown among physicists, we find it recessary to explain them in some detail. This is done in sect. 1, where proofs have been omitted in general, trying instead to empha- 
size the notational conventions, definitions and main equations used in the rest of this work. The reader is referred to the original papers $[4,5,17,18,6]$ for a more complete presentation of the subject. This techniques are extended in sects. 2 and 3 , which contain the main results of this chapter, so as to give a functional $W(R, L, U)$ depending on a single octet of meson fields. For the case of four-dimensional space-time this functional is actually constructed and compared with Witten's result in sect. 4.

\subsection{Differential geometry and the effective functional}

$$
W\left(R, L, g_{R}, g_{L}\right)
$$

In what follows it will be more convenient to write the equations in terms of the left and right gauge fields $L$ and $R$ :

$$
V_{+} \equiv V+\gamma_{5} A \equiv \dot{r} R+\hat{\imath} L
$$

with $R=V+A$ and $L=V-A . \hat{r}$ and $\hat{l}$ are the right and left projection operators:

$$
\hat{f}=\frac{1}{2}\left(1+\gamma_{8}\right) \quad, \quad \hat{l}=\frac{1}{2}\left(1-\gamma_{5}\right)
$$

Defining left and right spinors $\psi_{L}=\hat{\imath} \psi$ and $\psi_{R}=\hat{r} \psi$ the lagrangian (1) can be split:

$$
\mathcal{L}=i \bar{\psi}_{R} \gamma^{\mu}\left(\partial_{\mu}-i \lambda_{k} R_{\mu}^{k}\right) \psi_{R}+(R \leftrightarrow L)
$$

A general transformation $g(x) \in G \times G$ will be written:

$$
g=e^{\beta+7 s \alpha}=e^{p(\theta+\alpha)+i(\beta-\alpha)}=\hat{r} g_{R}+\hat{l} g_{L}
$$

with $g_{R}=e^{v_{n}}=e^{\theta+a}$ and $g_{L}=e^{u_{L}}=e^{\beta-\alpha}$. From (1.5), (1.9) and (1.11) it follows that the gauge fields $R \equiv A_{R}$ and $L \equiv A_{L}$ transform independently 
of each other:

$$
\begin{aligned}
& A_{H} \rightarrow g_{H}^{-1} A_{H} g_{H}+g_{H}^{-1} d g_{H} \quad, \quad H=L, R \\
& F_{H} \rightarrow g_{H}^{-1} F_{H} g_{H}
\end{aligned}
$$

where $F_{H}=d A_{H}+A_{H}^{2}$ ( $d$ is the exterior differential operator, and it is understood that $A_{H}^{2}$ stands for $A_{H} \wedge A_{H}$ ).

The left and right currents have anomalous covariant divergences:

$$
D^{\mu} J_{\mu k}^{H}=-G_{h}^{H}\left(A_{H}\right) \quad \text { with } \quad J_{\mu h}^{H}=\bar{\psi}_{H} \gamma_{\mu} \lambda_{k} \psi_{H}
$$

The functions $G_{k}^{H}$ were obtained explicitly by Gross and Jackiw [2], and in fact

$$
G_{k}^{H} \equiv \eta_{H} G_{h} \quad, \quad \eta_{R}=-\eta_{L}=1
$$

These functions can also be obtained by differential geometry methods without the use of Feynman diagrams [4]. To this end we have to define a number of differential geometric objects. Given the left-right symmetry expressed in eqs. (1.12) and (1.14), in what follows the index $H$ will not be written. The frst object is the $n$th Chern character $\Omega_{2 n}$ :

$$
\Omega_{2 n}(A)=\operatorname{tr} F^{n}
$$

The form $\Omega_{2 n}$ is closed $\left(d \Omega_{2 n}=0\right)$, and as a consequence can be locally expressed as the total differential of a form $\omega_{2 n-1}^{0}(A)$. An explicit expression for $\omega_{2 n-1}^{0}$ is obtained by considering a 1-parameter family of gauge fields $A_{t}$. Under an ordinary variation $\delta t, \Omega_{2 n}$ experiences a variation given by

$$
\delta \Omega_{2 n}\left(A_{t}\right)=n d \operatorname{tr}\left(\delta A_{t} F_{t}^{n-1}\right)
$$


Choosing the parametrization $A_{t}=t A,(1.16)$ can be readily integrated and we get for $w_{2 n-1}^{0}$

$$
\omega_{2 n-1}^{0}(A)=n \int_{0}^{1} \delta t \operatorname{tr}\left(A F_{t}^{n-1}\right)
$$

with $F_{\mathrm{l}}=d A_{\mathrm{l}}+A_{\mathrm{i}}^{2}=t d A+t^{2} A^{2}=t F+t(t-1) A^{2}$.

Since $\omega_{z n-1}^{0}$ is defined by $d \omega_{2 n-1}^{0}=\Omega_{2 n}$, we could add a total differential to (1.17). This fact will be exploited later in sects. 2 and 3.

$\Omega_{2 n}$ is invariant under gauge transformations. This allows the definition of a form $\omega_{2 n-2}^{1}(A, v)\left(g=e^{\nu}\right)$ as follows. From the definition of $\omega_{2 n-1}^{0}$,

$$
\delta_{v} \Omega_{2 n}(A)=\delta_{v} d \omega_{2 n-1}^{0}(A)=d\left(\delta_{v} \omega_{2 n-1}^{0}(A)\right)=0
$$

This mearis that $\delta_{v} \omega_{2 n-1}^{0}$ is closed and can be locally expressed as a total differential:

$$
\delta_{u} \omega_{2 n-1}^{0}(A)=d \omega_{2 n-2}^{1}(A, v)
$$

The form $\omega_{2 n-2}^{1}$ is important because it gives directly a solution to the WessZumino consistency conditions, and contains the anomalous divergences $G_{k}$ in $(2 n-2)$-dimensional space-time:

$$
K_{n} \omega_{2 n-2}^{1}(A, v)=v^{h} G_{k} \quad \text { with } \quad K_{n}=\frac{1}{n !} \frac{i^{n}}{(2 \pi)^{n-1}}
$$

(see appendix $A$ for an explicit expression for $\omega_{2 n-2}^{1}$.)

We can now proceed to the construction of an effective functional. We define formally

$$
W^{\prime}(A)=K_{n} \int_{D_{2 n-1}} \omega_{2 n-1}^{0}(A)
$$

where $D_{2 n-1}$ is a (2n-1)-dimensional disk which has as boundary space-time compactified to a sphere $S^{2 n-2}$.Under a gauge transformation

$$
\delta_{v} W^{\prime}=K_{n} \int_{D_{2 n-1}} \delta_{v} \omega_{2 n-1}^{0}(A)=K_{n} \int_{D_{2 n-1}} d \omega_{2 n-2}^{1}(A, v)
$$




$$
=K_{n} \int_{S^{2 n-2}} \omega_{2 n-2}^{1}(A, v)=\int_{S^{2 n-2}} v^{k} G_{k}
$$

and $W^{\prime}$ satisfies

$$
\left.\frac{\delta W^{\prime}}{\delta v^{k}}\right|_{u=0}=G_{k}
$$

This definition of $W^{\prime}$ is not totally satisfactory, since although $\omega_{2 n-1}^{0}$ is a finite polynomial in the gauge fields, it is not a total differential, and when we try to express it as an integral over $(2 n-2)$-dimensional space-time, the integrand becomes nonlocal, ie., contains interactions of arbitrary high order. This problem can be fixed by considering the following property of $\omega_{2 n-1}^{0}$ :

$$
\begin{aligned}
T(g) \omega_{2 n-1}^{0}(A, F) & \equiv \omega_{2 n-1}^{0}\left(A_{g}, F_{g}\right) \\
& =\omega_{2 n-1}^{0}(A, F)+\omega_{2 n-1}^{0}\left(g^{-1} d g, 0\right)+d \alpha_{2 n-2}(A, g)(1
\end{aligned}
$$

with $A_{q} \equiv T(g) A=g^{-1} A g+g^{-1} \dot{d g}$ and $F_{q}=g^{-1} F g$.

In this expression $\omega_{2 n-1}^{0}\left(g^{-1} d g, 0\right)$ has a topological significance [10]. We will refer to it as $\Delta_{2 n-1}(g)$. An explicit expression follows easily from (1.17):

$$
\omega_{2 n-1}^{0}\left(g^{-1} d g, 0\right) \equiv \Delta_{2 n-1}(g)=(-1)^{n+1} n \beta(n, n) \operatorname{tr}\left(g^{-1} d g\right)^{2 n-1}
$$

where $\beta(n, n)$ is the $\beta$-function given by

$$
\beta(n, n)=\frac{\Gamma(n) \Gamma(n)}{\Gamma(2 n)}=\frac{(n-1) !(n-1) !}{(2 n-1) !}
$$

An expression for $\alpha_{2 n-2}$ in which it appears as a finite polynomial in $A$ and $V \equiv d g g^{-1}$ can be found in appendix $A$. Since $F$ is a function of $A$, we will write $\omega_{2 n-1}^{0}(A, F) \equiv \omega_{2 n-1}^{0}(A)$. From (1.12) we have the group property

$$
T(g) T(h)=T(h g)
$$


and we get the identity

$$
T(g) \omega_{2 n-1}^{0}(A)=T\left(h^{-1} g\right) T(h) \omega_{2 n-1}^{0}(A)=T\left(h^{-1} g\right) \omega_{2 n-1}^{0}\left(A_{h}\right)
$$

In other words, $T(g) \omega_{2 n-1}^{0}$ is invariant under the transformation $A \rightarrow A_{h}$, $g \rightarrow h^{-1} g$. If we define

$$
W(A, g)=K_{n} \int_{D_{2 n-1}} \omega_{2 n-1}^{0}(A)-T(g) \omega_{2 n-1}^{0}(A)
$$

by (1.27) we have

$$
\left.\frac{\delta W}{\delta v^{k}}\right|_{v=0}=\left.\frac{\delta W^{\prime}}{\delta v^{k}}\right|_{v=0}=G_{k}(A)
$$

and by $(1.24) W(A, g)$ can be written

$$
\begin{aligned}
W(A, g) & =-K_{n} \int_{D_{2 n-1}} \Delta_{2 n-1}(g)+d \alpha_{2 n-2}(A, g) \\
& =-K_{n} \int_{D_{2 n-1}} \Delta_{2 n-1}(g)-K_{n} \int_{S^{2 n-2}} \alpha_{2 n-2}(A, g)
\end{aligned}
$$

Now the gauge fields appear only in the second integral, which is explicitly a finite polynomial in $A$ and $V \equiv d g g^{-1}$ as given by (A.3). There exists only a finite number of vertices containing the gauge fields and $g$. Since $\Lambda_{2 n-1}(g)$ cannot be written as a total differential, we still have an infinite number of vertices, but only in the field $g$.

Taking into account the signs given by (1.14), the complete functional is

$$
W\left(R, L, g_{R}, g_{L}\right)=W\left(R, g_{R}\right)-W\left(L, g_{L}\right)
$$

where $g_{R}(x)=e^{i \ell_{n}(x)}$ and $g_{L}(x)=e^{\left.i \xi_{L}(x)\right)}$ are elements of $G$.

\subsection{The functional $W(R, L, U)$}

In this section we modify the functional $W\left(R, L, g_{R}, g_{L}\right)$ so as to make it depend on a single field $U(x)(U \in G)$ instead of $g_{R}$ and $g_{L}$. To this end we 
define

$$
\omega_{2 n-1}^{0}(R, L) \equiv \omega_{2 n-1}^{0}(R)-\omega_{2 n-1}^{0}(L)
$$

and notice that by $(1.28),(1.31)$ and (1.32) the effective functional can be written as

$$
W\left(R, L, g_{R}, g_{L}\right)=K_{n} \int_{D_{2 n-1}} \omega_{2 n-1}^{0}(R, L)-T\left(g_{R}, g_{L}\right) \omega_{2 n-1}^{0}(R, L)
$$

Here $T\left(g_{R}, g_{L}\right)$ defines the action of an element $g=g_{R} \hat{r}+g_{L} \hat{l} \in G \times G$ :

$$
T\left(g_{R}, g_{L}\right) \omega_{3 n-1}^{0}(R, L) \equiv \omega_{2 n-1}^{0}\left(R_{\rho R}, L_{Q_{L}}\right)
$$

If we define the $n$th Chern character for the whole group $G \times G$ as

$$
\boldsymbol{\Omega}_{2 n}(R, L)=\Omega_{2 n}(R)-\Omega_{2 n}(L)
$$

then $w_{2 n-1}^{0}(R, L)$ as given by $(1.32)$ is a solution to the equation

$$
\Omega_{2 n}(R, L)=d \omega_{2 n-1}^{0}(R, L)
$$

In the next section we will show that another solution $\bar{\omega}_{2 n-1}^{0}(R, L)$ to (1.36) can always be found, such that it is invariant under vector gauge transformations:

$$
\delta_{\beta} \bar{\omega}_{2 n-1}^{0}(R, L)=0
$$

By (1.36) it can only differ from (1.32) by a total differential:

$$
\ddot{\omega}_{2 n-1}^{0}(R, L)=\omega_{2 n-1}^{0}(R, L)+d S_{2 n-2}(R, L)
$$

If we consider the functional

$$
\bar{W}\left(R, L g_{R}, g_{L}\right)=K_{n} \int_{D_{2 n-1}} \omega_{2 n-1}^{0}(R, L)-T\left(g_{R}, g_{L}\right) \dot{\nu}_{2 n-1}^{0}(R, L)
$$


and use Stokes theorem $\left(\partial D_{2 n-1}=S^{2 n-2}\right)$, we can see that $\bar{W}$ is related to $W$ by

$$
\bar{W}\left(R, L g_{R}, g_{L}\right)=W\left(R, L, g_{R}, g_{L}\right)-K_{n} \int_{S^{2 n-2}} T\left(g_{R}, g_{L}\right) S_{2 n-2}(R, L)
$$

By the group property $T\left(g_{R}, g_{L}\right) T\left(h_{R}, h_{L}\right)=T\left(h_{R} g_{R}, h_{L} g_{L}\right)$, the last piece of $(1.40)$ is invariant under the local transformation

$$
R \rightarrow R_{h_{R}}, \quad L \rightarrow L_{h_{L}} ; \quad g_{R} \rightarrow h_{R}^{-1} g_{R}, \quad g_{L} \rightarrow h_{L}^{-1} g_{L}
$$

This means

$$
\frac{\delta \bar{W}}{\delta v_{H}^{k}}=\frac{\delta W}{\delta v_{H}^{k}}=G_{k}^{H}, \quad H=L, R
$$

Also, in the next section $S_{2 n-2}(R, L)$ will be calculated explicitly as a finite polynomial in the gauge fields. $\bar{W}$ is thus local in $R$ and $L$, and by (1.42) is a satisfactory effective functional.

Now we use the group property to combine $g_{R}$ and $g_{L}$ into one single field:

$$
\begin{aligned}
T\left(g_{R}, g_{L}\right) \bar{\omega}_{2 n-1}^{0}(R, L) & =T\left(g_{R}, g_{R}\right) T\left(e, g_{L} g_{R}^{-1}\right) \bar{\omega}_{2 n-1}^{0}(R, L) \\
& =T\left(g_{R}, g_{R}\right) \bar{\omega}_{2 n-1}^{0}\left(R, L_{2 L g_{n}^{-1}}\right)=\bar{\omega}_{2 n-1}^{0}\left(R, L_{2 L g_{n}^{-1}}\right) \\
& =T\left(e, g_{L}, g_{R}^{-1}\right) \bar{\omega}_{2 n-1}^{0}(R, L)
\end{aligned}
$$

since $T\left(g_{R}, g_{L}\right)$ is a vector gauge transformation and has no effect on $\bar{\omega}_{2 n-1}^{0}$. If we define $U \equiv g_{L} g_{R}^{-1} \in G$, we have

$$
T\left(g_{R}, g_{L}\right) \tilde{\omega}_{2 n-1}^{0}(R, L)=T(e, U) \tilde{\omega}_{2 n-1}^{0}(R, L)
$$

where $e$ is the identity element of $G$, and from (1.41) $U$ transforms as

$$
U \rightarrow h_{L}^{-1} U h_{R}
$$


We can now define the effective functional $W(R, L, U)$ :

$W(R, L, U) \equiv \tilde{W}\left(R, L, g_{R}, g_{L}\right)=K_{n} \int_{D_{2 n-1}} \omega_{2 n-1}^{0}(R, L)-T(e, U) \tilde{\omega}_{2 n-1}^{0}(R, L)$

By (1.38) anc (1.24) the last piece can be written

$$
T(e, U) \bar{\omega}_{2 n-1}^{0}=\omega_{2 n-1}^{0}(R, L)-\Lambda_{2 n-1}(U)-d \alpha_{2 n-2}(L, U)+d S_{2 n-2}\left(R, L_{U}\right)
$$

and the final expression is

$$
W(R, L, U)=K_{n} \int_{D_{2 n-1}} \Delta_{2 n-1}(U)+K_{n} \int_{S^{2 n-2}} \alpha_{2 n-2}(L, U)-S_{2 n-2}\left(R, L_{U}\right)
$$

where $L_{U}=U^{-1} L U+U^{-1} d U$, and $U(x)=e^{i \ell(x)}$ is an element of $G$.

$W(R, L, U)$ is the Wess-Zumino effective action. The first integral contains the Wess-Zumino term and the second one is the result of its gauging. Notice that, by (1.37), $S_{2 n-2}$ has the following behavior under vector gauge transformations:

$$
\delta_{\rho} \omega_{2 n-1}^{0}(R, L)=-\delta_{\rho} d S_{2 n-2}
$$

and it follows

$$
\frac{\delta}{\delta \beta^{k}}\left(W(R, L, U)+\int_{S^{2 n-2}} S_{2 n-2}(R, L)\right) \equiv \frac{\delta \dot{W}(R, L, U)}{\delta \beta^{k}}=\bar{G}_{k}^{V}(R, L)=0
$$

i.e. the addition of $S_{2 n-2}(R, L)$ to the lagrangian removes the anomaly from the vector current. $S_{2 n-2}(R, L)$ is thus equivalent, in four dimensions, to the counterterm used by Bardeen [5| to conserve the vector current and we will refer to it simply as 'the counterterm'. An expression for $S_{2 n-2}(R, L)$ valid for any $n$ is derived in the next section. 
It is an interesting fact that the counterterm, which was first used by Bardeen to get the conserved vector current form of the anomaly, appears in our method as an essential ingredient in the construction of $W(R, L, U)$, which by (1.42) gives the anomaly in the left-right symmetric form.

\subsection{The counterterm}

To find solutions to the equation

$$
d w_{2 n-1}^{0}=\Omega_{2 n}(R, L)
$$

in a systematic way, it is convenient to express $\Omega_{2 n}$ as a function of $V_{+}=$ $\hat{r} R+\hat{l} L:$

$$
\Omega_{2 n}\left(V_{+}\right)=\frac{2}{D} \operatorname{tr}\left(\gamma_{s} F_{+}^{n}\right) \equiv \Omega_{2 n}(R, L)
$$

where $F_{+}=d V_{+}+V_{+}^{2}=\left(d R+R^{2}\right) \hat{f}+\left(d L+L^{2}\right) \hat{l}$, and $D \times D$ is the dimension of the Dirac matrices. The equivalence of (1.35) and (1.51) follows from the fact that $\hat{f}$ and $\hat{l}$ are projection operators and $\gamma_{5}=\hat{\boldsymbol{r}}-\hat{l}$.

We consider a 2-parameter family of onc-forms :

$$
A_{\lambda \mu}=\lambda V_{+}+\mu V_{-}
$$

with $V_{-}=V-\gamma_{s} A=\hat{\gamma} L+\hat{l} R$. An ordinary variation $(\delta \lambda, \delta \mu)$ of the parameters induces a variation in $\Omega_{2 n}$ given by

$$
\delta \Pi_{2 n}\left(A_{\lambda \mu}\right)=\frac{2 \pi}{D} d \operatorname{tr}\left(\gamma_{5} \delta A_{\lambda_{\mu}} F_{\lambda \mu}^{n-1}\right)
$$

where $\delta A_{\lambda \mu}=\delta \lambda V_{+}+\delta \mu V_{-}$and $F_{\lambda \mu}=d A_{\lambda \mu}+A_{\lambda \mu}^{2}$.

Now we observe that $\Omega_{2 n}\left(A_{00}\right)=0$ and

$$
\Omega_{2 n}\left(A_{10}\right)=\Omega_{2 n}\left(V_{+}\right)
$$




$$
\Omega_{2 n}\left(A_{01}\right)=\Omega_{2 n}\left(V_{-}\right)=-\Omega_{2 n}\left(V_{+}\right)
$$

From this it follows that $\Omega_{2 n}\left(V_{+}\right)$can be expressed as the differential of a line integral

$$
\Omega_{2 n}\left(V_{+}\right)=\frac{2 n}{D} d \int_{(a) \text { or }(b)} \operatorname{tr}\left(\gamma_{5} \delta A_{\lambda \mu} F_{\lambda \mu}^{n-1}\right)=\frac{n}{D} d \int_{(c)} \operatorname{tr}\left(\gamma_{5} \delta A_{\lambda \mu} F_{\lambda \mu}^{n-1}\right)
$$

where the integrals are along the oriented paths (a), (b) or (c) shown in fig. 1. Each path gives a different solution to eq. (1.36). In particular, if we integrate along the straight line $\mu=0$ we get the solution (1.32):

$$
\begin{aligned}
\frac{2 n}{D} \int_{0}^{1} \delta \lambda \operatorname{tr}\left(\gamma_{5} V_{+} F_{+}^{n-1}\right) & =n \int_{0}^{1} \delta \lambda \operatorname{tr}\left(R F_{R}\right)^{n-1}-(R \leftrightarrow L) \\
& =\omega_{2 n-1}^{0}(R)-\omega_{2 n-1}^{0}(L)
\end{aligned}
$$

The same result is obtained by integration along the line $\lambda=0$. It is easy to show (see appendix B) that the solution obtained when we integrate along $\mu+\lambda=1$ is invariant under vector gauge transformations, i.e.

$$
\bar{\omega}_{2 n-1}^{0}(R, L)=\frac{n}{D} \int_{(0,1) \text { along } \lambda+\mu=1}^{(0,1)} \operatorname{tr}\left(\gamma_{B} \delta A_{\lambda \mu} F_{\lambda \mu}^{n-1}\right)
$$

From (1.56), (1.57) and (1.38) an expression in which $d S_{2 n-2}(R, L)$ is represented by a closed line integral is readily obtained (see fig. 2)

$$
\frac{n}{D} \int \operatorname{tr}\left(\gamma_{3} \delta A_{\lambda \mu} F_{\lambda \mu}^{n-1}\right)=\bar{\omega}_{2 n-1}^{0}-\omega_{2 n-1}^{0}=d S_{2 n-2}(R, L)
$$

(Here all the integrals are along straight lines.)

A more convenient expression giving directly $S_{2 n-2}(R, L)$ is constructed from (1.58) in appendix $B$. The result is

$$
S_{2 n-2}(R, L)=\frac{n(n-1)}{D} \iint \operatorname{Str}\left(\gamma_{5} V_{-} V_{+} F_{\lambda \mu}^{n-2}\right)
$$


where the integral is over the interior of the triangle represented in fig. 2. The following property is useful in the actual evaluation of (1.59): given the trace of a monomial containing $\gamma_{5}$ and the fields $V_{ \pm}$and/or $F_{ \pm}$, the reversal of all the signs in $V_{ \pm}$and $F_{ \pm}$reproduces the original expression with opposite sign. For instance

$$
\operatorname{tr}\left\{\gamma_{5} V_{+} V_{-} F_{-}^{2}\right\}=-\operatorname{tr}\left\{\gamma_{5} V_{-} V_{+} F_{+}^{2}\right\}
$$

\subsection{An application}

In this section the functional $W(R, L, U)$ is actually constructed for the case $n=3$, i.e. four-dimensional space-time. $W$ contains three functions that have to be evaluated:

$$
W(R, L, U)=K_{3} \int_{D_{3}} \Delta_{5}(U)+K_{3} \int_{S^{4}} \alpha_{4}(L, U)-S_{4}\left(R, L_{U}\right)
$$

From (1.25)

$$
\Lambda_{8}=\frac{1}{10} \operatorname{tr}\left(U^{-1} d U\right)^{5}
$$

and from (A.3)

$$
\begin{aligned}
\alpha_{1}(L, U)= & -\frac{1}{2} \operatorname{tr}\left\{\left(d U U^{-1}\right)\left(L d L+d L L+L^{s}\right)\right. \\
& \left.-\frac{1}{2}\left(d U U^{-1}\right) L\left(d U U^{-1}\right) L-\left(d U U^{-1}\right)^{3} L\right\}
\end{aligned}
$$

$S_{1}$ is obtained from (1.59) as a function of the fields $V_{+}$and $V_{-}$:

$$
S_{4}=\frac{1}{4} \operatorname{tr}\left\{\gamma_{5} V_{-}\left(V_{+} F_{+}+F_{+} V_{+}-V_{+}^{3}\right)+\frac{1}{4} \gamma_{5} V_{-} V_{+} V_{-} V_{+}\right\}
$$

Using the fact that $f$ and $\hat{l}$ are projection operators and that in four dimensions $\operatorname{tr} \hat{r}=\operatorname{tr} \hat{l}=2, S_{4}$ may be written in terms of $R$ and $L$

$$
S_{4}(R, L)=\frac{1}{2} \operatorname{tr}\left\{(L R-R L)\left(F_{R}-F_{L}\right)+R^{3} L+R L^{3}+\frac{1}{2} L R L R\right\}
$$


In this last expression we have rrade the substitution

$$
L \rightarrow L_{U} \equiv U^{-1} L U+U^{-1} d U
$$

The normalization constant $K_{3}$ is given by (1.20)

$$
K_{3}=\frac{-i}{24 \pi^{2}}
$$

After collecting the terms arising from (1.61), (1.62) and (1.64) with (1.65), and making the substitution

$$
R d U^{-1} d L U-R d U^{-1} L d U \rightarrow-d R d U^{-1} L U
$$

(the left- and right-hand side of (1.67) differ by the total differential of $\left.R d U^{-1} L U\right)$ to get a more symmetrical expression we obtain

$$
W(R, L, U)=\frac{-i}{240 \pi^{2}} \int_{D_{6}} \operatorname{tr}\left(U^{-1} d U\right)^{5}-\frac{i}{48 \pi^{2}} \int_{S^{4}} Z
$$

where the 4 -form $Z$ is

$$
\begin{aligned}
Z= & -\operatorname{tr}\left\{U_{L}\left(L d L+d L L+L^{3}\right)-\left(U_{L}\right)^{3} L\right\}-\operatorname{tr}\{R \leftrightarrow L\} \\
& +\frac{1}{2} \operatorname{tr}\left(U_{L} L U_{L} L\right)-\frac{1}{2} \operatorname{tr}\{R \leftrightarrow L\}-\operatorname{tr}\left(U^{-1} L U R^{3}\right) \\
& +\operatorname{tr}\left(U R U^{-1} L^{3}\right)-\operatorname{tr}\left\{U^{-1} L U(R d R+d R R)\right\} \\
& +\operatorname{tr}\left\{U R U^{-1}(L d L+d L L)\right\}-\operatorname{tr}\left\{U R U^{-1} L U_{L} L\right\} \\
& -\operatorname{tr}\left\{U^{-1} L U R U_{R} R\right\}+\operatorname{tr}\left\{L d U U_{R} R U^{-1}\right\}+\operatorname{tr}\left\{R d U^{-1} U_{L} L U\right\} \\
& -\operatorname{tr}\left\{d L d U R U^{-1}\right\}+\operatorname{tr}\left\{d R d U^{-1} L U\right\} \\
& +\frac{1}{2} \operatorname{tr}\left\{R U^{-1} L U R U^{-1} L U\right\}
\end{aligned}
$$

Here $U_{L}$ stands for $d U U^{-1}$ and $U_{R}$ for $U^{-1} d U$. 
Eq. (1.69) is our final result. To compare it with the expression obtained by Witten $[10]$ we must notice that $A_{R} \equiv-i R$ and $A_{L} \equiv-i L$ due to a different convention in the definition of the covariant derivative. We find some minor differences with Witten's effective runctional, which he calls $\bar{\Gamma}$, which does not seem to have the prescribed transformation properties. If one makes the following changes in $Z_{\mu \nu a \beta}$ (of $\bar{\Gamma}$ ):

(i) Delete the coefficient $1 / 2$ in front of $A_{\mu L} A_{\nu L} U A_{\alpha R} A_{\beta R} U^{-1}$;

(ii) Interchange $K \leftrightarrow L$ in the second term of the third line:

$$
A_{\mu L} U^{-1}\left(\partial_{\nu} A_{\alpha R}\right) \partial_{\beta} U \rightarrow A_{\mu R} U^{-1}\left(\partial_{\nu} A_{\alpha L}\right) \partial_{\beta} U
$$

(iii) Add $i A_{\mu L} A_{\nu L} A_{\alpha L}$ to the expression $\left(\partial_{\mu} A_{\nu L}\right) A_{\alpha L}+A_{\mu L}\left(\partial_{\nu} A_{\alpha L}\right)$ in the second line; then the final expression transforms correctly, and the relation with (1.69) is

$$
\tilde{\Gamma}=W-\frac{i}{48 \pi^{2}} \int_{S^{4}} \operatorname{tr}\left(F_{L} U F_{R} U^{-1}\right)-d \operatorname{tr}\left(L U d R U^{-1}\right)
$$

The last term, being a total differential, vanishes upon integration by parts, and the first term is invariant under both vector and axial vector gauge transformations and is therefore irrelevant.

Witten has established the a priori quantization of the Wess-Zumino action in the sense that $W$ has to be multiplied by an integer which he has found to be equal to the number of colors $N_{c}$ (10). Taking this into account, the anomalous contribution of the field $U$ to the baryon current can be calculated directly from (1.89). To this end, consider a local transformation of the fundamental spinor fields in the lagrangian (1.1) given by

$$
\psi(x) \rightarrow \exp \left(i \frac{v^{0}(x)}{N_{c}}\right) \psi(x)
$$


where $1 / N_{\epsilon}$ is the baryon number of a fermion (quark). By (1.3) and (1.4) this corresponds to a generator $\lambda_{0}$ given by

$$
\lambda_{0}=\frac{1}{N_{\epsilon}}
$$

where 1 is the $f \times f$ unit matrix ( $f$ is the number of flavors).

Then the anomalous vector baryon current is easily computed:

$$
\begin{aligned}
* J_{0}^{V} & =* J_{0}^{R}+* J_{0}^{L}=N_{c}\left(\frac{\delta W}{\delta R^{0}}+\frac{\delta W}{\delta L^{0}}\right)_{R=L=0} \\
& =\frac{-i N_{c}}{48 \pi^{2}} \operatorname{tr}\left\{\frac{1}{N_{c}}\left(i U_{L}^{3}+i U_{R}^{3}\right)\right\}=\frac{1}{24 \pi^{2}} \operatorname{tr}\left(U^{-1} d U\right)^{s}
\end{aligned}
$$

since $\operatorname{tr}\left(U_{L}^{S}\right)=\operatorname{tr}\left(U_{R}^{S}\right) \equiv \operatorname{tr}\left(U^{-1} d U\right)^{3}$. Note that $J_{D}^{A}$ (anomalous) vanishes identically.

Eq. (1.73) coincides with the expression given by Witten $[10]$ by analogy with the electromagnetic anomalous current. The addition of the $U(1)$ generator $\lambda_{0}$ to the generators of $S U(3)$ increases the number of mesons to nine, the new one being a pseudoscalar (singlet). From the fact that $\Pi_{s}(S U(2))=Z$ it is clear that all the meson fields but the pion triplet can be set equal to zero, and one still gets an anomalous baryon number contribution $B_{0}[19]$ :

$$
B_{0}=\frac{1}{24 \pi^{2}} \int_{S \text { paea }} \operatorname{tr}\left(U^{-1} d U\right)^{3}
$$




\section{Figure captions}

1. The $(\lambda, \mu)$ parameter space and paths associated with different solutions to eq. (1.36)

2. Domain of integration for $S_{2 n-2}$ 


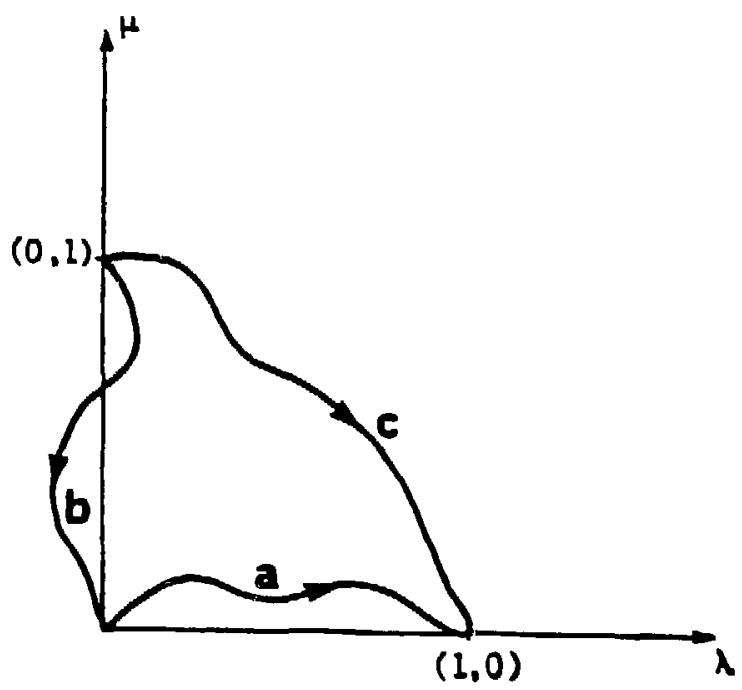

Figure 1

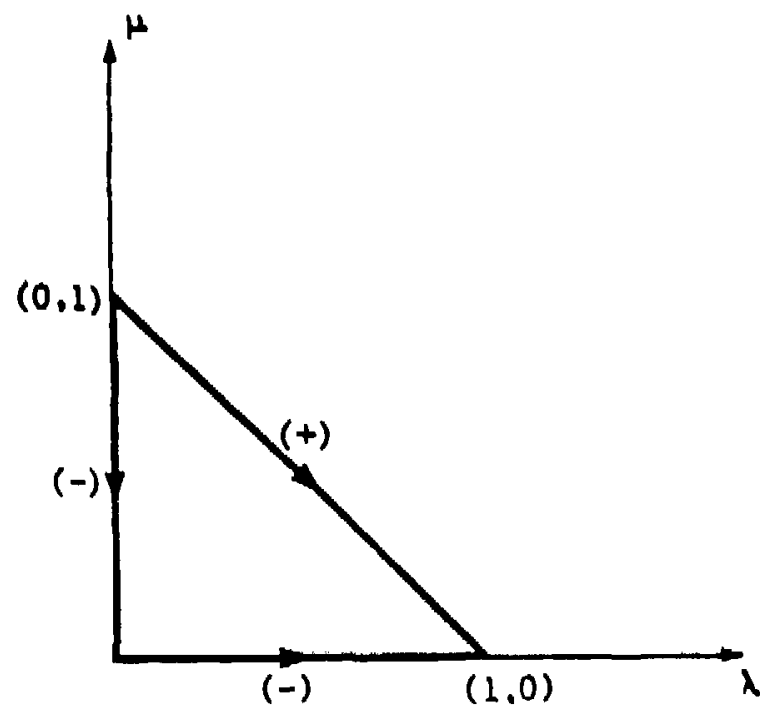

Figure 2 


\section{WKB METHOD FOR BOSE-FERMI}

\section{QUANTUM-MECHANICAL SYSTEMS}

The purpose of this chapter is to develop a WKB approximation for the kernel of a mixed (Bose-Fermi) quantum-mechanical system.This approximation is used in chapter 3 to compute the index of the Dirac operator which, as we shall see, can be written as the supertrace of the kernel for the appropriate supersymmetric system.

We shall consider the computation of the kernel for a quantum mechanical Hamiltonian $\hat{H}$ which is a function of bosonic operators $\hat{t}^{\mu}$ and $\hat{p}_{\mu}$ and fermionic operators $\hat{\bar{\theta}}_{a}$ and $\hat{\theta}_{a}$. These operators satisfy

$$
\left[\hat{p}_{\mu}, \hat{x}^{\nu}\right]=\frac{\hbar}{i} \delta_{\mu}^{\nu}, \quad\left\{\hat{\bar{\theta}}_{a}, \hat{\theta}_{b}\right\}=\hbar \delta_{a b}
$$

and any bosonic operator commutes with any fermionic operator.

The canonical fermionic momenta are conventionally defined by

$$
\hat{\pi}_{a}=-i \hat{\bar{\theta}}_{a}
$$

and therefore

$$
\left\{\hat{\pi}_{a}, \hat{\theta}_{b}\right\}=\frac{\hbar}{i} \delta_{a b}
$$

In the classical deseription of the system $x^{\mu}$ and $p_{\mu}$ are ordinary real variables, and $\bar{\theta}_{a}, \theta_{a}$ and $\pi_{a}$ are anticommuting or Grassman variables.

Some concepts of classical mechanics, generalized to systems with fermionic variables, are presented in section 1 . These concepts are used in section 2 to give a WKB formula for the kernel of a general quantum mechanical Hamiltonian with bosonic and fermionic operators. In the case of a purely bosonic 
system this formula reduces to the well-known expression $[23,24]$.

$$
K\left(x_{t}, x_{0} ; t\right) \sim \sqrt{D} \exp \left(\frac{i}{\hbar} S\left(x_{t}, x_{0} ; t\right)\right)
$$

where $S$ is the classical Hamilton-Jacobi function and $D$ is the Van Vleck determinant. Some ordering problems arising when fermionic variables are present are analyzed in detail.

Section 3 is dedicated to the computation of traces and supertraces of the evolution operator in the WKB approximation, and a general analysis of the validity of this approximation is presented. This analysis is used in chapter 3 to show that, in the case of the supersymmetric quantum mechanical system used to prove the index theorem, the WKB formula is exact.

\subsection{Classical mechanics with fermionic variables}

To simplify the notation we shall use $Q$ instead of $(x, \theta)$ and $P$ instead of $(p, \pi)$. Omitting obvious indices, the action functional is

$$
\left.I=\int_{0}^{t} \dot{Q} P-X(Q, P)\right) d t^{\prime}
$$

The equations of motion are obtained by imposing $\delta I=0$ under any arbitrary variation with $\delta Q_{0}=\delta Q_{1}=0$. The result is

$$
\delta \mathcal{H}-\dot{Q} \delta P+\delta Q \dot{P}=0
$$

which can also be written

$$
\dot{Q}_{k}=\sigma(k) \frac{\partial H}{\partial P_{k}} \quad, \quad \dot{P}_{k}=-\frac{\partial H}{\partial Q_{k}}
$$

where

$$
\sigma(k)= \begin{cases}+1 & \text { if } Q_{k} \text { is bosonic } \\ -1 & \text { if } Q_{k} \text { is fermionic }\end{cases}
$$


Note that the order in which factors are written is important because of the presence of Grassman variables.

We shall now describe some functions which will be used in the WKB construction of the kernel. These are the modified Hamilton-Jacobi function and the generalized Van Vleck superdeterminant. Recall that the HamiltonJacobi function or "classical" action is defined by [25]

$$
S\left(Q_{t}, Q_{0} ; t\right) \equiv \int_{0}^{t}(\dot{Q} P-\not H) d t^{\prime}
$$

where the integration is along the classical trajectory from $\left(Q_{0} ; 0\right)$ to $\left(Q_{t} ; t\right)$. Under a variation of $t, Q_{t}$ and $Q_{0}$ the change in the classical action is

$$
\delta S=\delta Q_{t} P_{t}-\delta Q_{0} P_{0}-\not \delta \delta t
$$

This last expression gives the initial and final momenta as functions of $Q_{0}, Q_{t}$ and $t$ once $S$ is known

$$
P_{t}=\frac{\partial S}{\partial Q_{t}}\left(Q_{t}, Q_{0} ; t\right), P_{0}=-\frac{\partial S}{\partial Q_{0}}\left(Q_{t}, Q_{0} ; t\right)
$$

and contains the Hamilton-Jacobi equation

$$
\frac{\partial S}{\partial t}+x\left(Q_{t}, \frac{\partial S}{\partial Q_{1}}\right)=0
$$

The generalization of the Van Vleck determinant [22] to a system with bosonic and fermionic variables is given (up to a sign) by the superdeterminant $[26 \mid$ of the supermatrix of second derivatives of the classical action with respect to initial and final coordinates

$$
\begin{aligned}
& S_{i j} \equiv \frac{\partial^{2} S}{\partial Q_{t i} \partial Q_{0 j}}, D=(-)^{N} \operatorname{Sdet}\left|S_{i j}\right| \\
& i, j: 1, \ldots, N
\end{aligned}
$$


By differentiating the Hamilton-Jacobi equation and using eq. (2.11) and the following property of the superdeterminant $[26]$

$$
\delta(\operatorname{Sdet} M)=\operatorname{Sdet} M \cdot \operatorname{Str}\left(M^{-1} \delta M\right)
$$

where $S t r$ is the supertrace, a continuity equation for $D$ is easily obtained

$$
\frac{\partial D}{\partial t}+\frac{\partial}{\partial Q_{t k}}\left(D \frac{\partial H}{\partial P_{1 k}}\left(Q_{t}, \frac{\partial S}{\partial Q_{t}}\right)\right)=0
$$

A derivation of this equation together with the definitions of supertrace and superdeterminant is presented in Appendix C.

It often happens, when one considers a system with fermionic variables, that the classical action $S\left(Q_{t}, Q_{0}, t\right)$ is not defined because there is no classical trajectory from $\left(Q_{0}, 0\right)$ to $\left(Q_{t}, t\right)$. As a simple example consider the following Hamiltonian

$$
H(\theta, \pi)=i \pi \theta
$$

The equations of motion are

$$
\dot{\theta}=-\frac{\partial H}{\partial \pi}=-i \theta, \dot{\pi}=-\frac{\partial H}{\partial \theta}=i \pi
$$

with solutions

$$
\pi_{t}=\pi_{0} e^{i t}, \theta_{t}=\theta_{0} \epsilon^{-i t}
$$

Obviously, for given $t$, we cannot specify both $\theta_{0}$ and $\theta_{l}$, but we can instead fix $\theta_{0}$ and $\pi_{t}$. This fact motivates the consideration of certain modified classical actions $\bar{S}$ which are defined as Legendre transforms of $S$ with respect to a subset of variables: 


$$
\begin{gathered}
\dot{S}\left(Q_{t i}, P_{t j}, Q_{0} ; t\right) \equiv S\left(Q_{t i}, P_{t j}, Q_{0} ; t\right)-Q_{t j}\left(Q_{t i}, P_{t j}, Q_{0} ; t\right) P_{t j} \\
i: 1, \ldots, R \quad j: R+1, \ldots, N
\end{gathered}
$$

where now

$$
S\left(Q_{t i}, P_{t j}, Q_{0} ; t\right) \equiv \int_{0}^{t}(\dot{Q} P-\not \not) d t^{\prime}
$$

and the integral is along the classical trajectory from $\left(Q_{0} ; 0\right)$ to $\left(Q_{t i}, P_{t j} ; t\right)$. $\bar{s}$ satisfies

$$
\delta \bar{S}=\delta Q_{t i} P_{i i}-Q_{t j} \delta P_{t j}-\delta Q_{0} P_{0}-\not \delta \delta t
$$

which implies

$$
P_{t i}=\frac{\partial \tilde{S}}{\partial Q_{t i}}, Q_{t j}=-\sigma(j) \frac{\partial \dot{S}}{\partial P_{t j}}, P_{0}=-\frac{\partial \bar{S}}{\partial Q_{0}}
$$

and the corresponding Hamilton-Jacobi equation is

$$
\frac{\partial \bar{s}}{\partial t}+*\left(Q_{t i},-\sigma(j) \frac{\partial \tilde{S}}{\partial P_{t j}}, \frac{\partial \bar{s}}{\partial Q_{t i}}, P_{t j}\right)=0
$$

The generalized Van Vleck superdeterminant is

$$
\begin{gathered}
\tilde{D} \equiv(-)^{N} \operatorname{Sdet}\left\|\begin{array}{c}
\frac{\partial^{2} \dot{S}}{\partial Q_{t i} \partial Q_{0 k}} \\
\frac{\partial^{2} \bar{S}}{\partial P_{t j} \partial Q_{0 k}}
\end{array}\right\| \\
i: 1, \ldots, R \quad j: R+1, \ldots, N \quad k: 1, \ldots, N
\end{gathered}
$$

and satisfies the following continuity equation

$$
\frac{\partial \bar{D}}{\partial t}+\frac{\partial}{\partial Q_{t i}}\left(\bar{D} \frac{\partial H}{\partial P_{t i}}\right)-\sigma(j) \frac{\partial}{\partial P_{t j}}\left(\bar{D} \frac{\partial H}{\partial Q_{t j}}\right)=0
$$

In practice, it will be particularly useful to Legendre transform only with respect to the fermionic variables. The reason is that for most of the cases of 
interest there exists a classical trajectory specified by the initial coordinates together with the final fermionic momenta and bosonic coordinates. In this case

$$
\begin{gathered}
S^{\prime}\left(x_{t}, \bar{\theta}_{t}, x_{0}, \theta_{0} ; t\right) \equiv S\left(x_{t}, \bar{\theta}_{t}, x_{0}, \theta_{0} ; t\right)-i \bar{\theta}_{t} \theta_{t} \\
D^{\prime}=(-)^{N} \operatorname{Sdet}\left\|\begin{array}{ll}
\frac{\partial^{2} S^{\prime}}{\partial x_{t} \partial x_{0}} & \frac{\partial^{2} S^{\prime}}{\partial x_{t} \partial \theta_{0}} \\
i \frac{\partial^{2} S^{\prime}}{\partial \bar{\theta}_{t} \partial x_{0}} & i \frac{\partial^{2} S^{\prime}}{\partial \bar{\theta}_{t} \partial \theta_{0}}
\end{array}\right\|
\end{gathered}
$$

where we have used $\bar{\theta}$ instead of $\pi=-i \bar{\theta}$.

\subsection{WKB expression for the kernel}

Given a quantum mechanical Hamiltonian

$$
\hat{H}=K\left(\hat{x}^{\mu}, \hat{p}_{\mu}, \hat{\theta}_{a}, \hat{\theta}_{a}\right)
$$

the kernel $K\left(x_{1}, \theta_{1}, x_{0}, \theta_{0} ; t\right)$ is defined by

$$
\Psi\left(x_{1}, \theta_{1} ; t\right)=\int d x_{0} \int_{0_{0}} K\left(x_{t}, \theta_{t}, x_{0}, \theta_{0} ; t\right) \Psi\left(x_{0}, \theta_{0} ; 0\right)
$$

where the wave function $\Psi$ satisfies Schrödinger's equation

$$
\mathcal{H}\left(x^{\mu}, \frac{n}{i} \partial_{\mu}, \frac{\partial}{\partial \theta_{a}}, \theta_{a}\right) \Psi+\frac{n}{i} \frac{\partial \Psi}{\partial t}=0
$$

and is normalized with respect to the following inner product $[27,28]$

$$
\left\langle\Psi_{1} \mid \Psi_{2}\right\rangle=\int d x \int_{1 \overline{0}} e^{\overline{0} \cdot N_{0}} \Psi_{1}^{*}(x, \bar{\theta}) \Psi_{2}(x, \theta)
$$

An explanation of the conventions used in the integration over Grassman variables is given in Appendix D. Complex conjugation takes $\theta_{a}$ into $\bar{\theta}_{a}$ and reverses the order of all anticommuting variables. 
Note that, with respect to the above inner product, $\hat{p}_{\mu}=(\hbar / i) \partial_{\mu}$ and $\hat{x}^{\mu}=x^{\mu}$ are hermitian operators, and $\hat{\bar{\theta}}_{a}=\partial / \partial \theta_{a}$ and $\hat{\theta}_{a}=\theta_{a}$ are hermitian conjugates of each other.

Equations (2.28) and (2.29) imply that $K$ satisfies

$$
\mathcal{H}\left(x_{t}^{\mu}, \frac{\hbar}{i} \frac{\partial}{\partial x_{t}^{\mu}}, \frac{\partial}{\partial \theta_{t a}}, \theta_{t a}\right) K\left(x_{t}, \theta_{t}, x_{0}, \theta_{0} ; t\right)+\frac{\hbar}{i} \frac{\partial K}{\partial t}=0
$$

and

$$
\lim _{t \rightarrow 0} K\left(x_{1}, \theta_{l}, x_{0}, \theta_{0} ; t\right)=\delta\left(x_{t}-x_{0}\right) \delta\left(\theta_{t}-\theta_{0}\right)
$$

Using the notation of the preceding section, the kernel $\bar{K}\left(Q_{t i}, P_{t j}, Q_{0 k ;} t\right)$ is defined by

$$
\begin{aligned}
\bar{\Phi}\left(Q_{t i}, P_{t j} ; t\right) & =\int_{Q_{0}} \bar{K}\left(Q_{t i}, P_{t j}, Q_{0 k i} t\right) \Psi\left(Q_{0 k i} 0\right) \\
i & : 1, \ldots, R \\
j & : R+1, \ldots, N \\
k & : 1, \ldots, N
\end{aligned}
$$

where $\bar{\Phi}$ is the appropriate Fourier transform of $\Psi . \bar{K}$ is, up to a sign, the cortesponding Fourier transform of $K$. (See Appendix D for details.)

In what follows we shall obtain an approximate expression for $\bar{K}$. We shall assume that the "exact" kernel $\bar{K}$ is given, for small $t$, by an asymptotic expansion of the form

$$
\dot{K} \approx \bar{C} \exp \left(\frac{i}{\hbar} \bar{S}\right)\left(\tilde{A}_{0}+\hbar \bar{A}_{1}+\hbar^{2} \bar{A}_{2}+\ldots\right)
$$

where $\bar{C}$ is a constant and $\bar{S}$ has been defined in eq. (2.19). The WKB 
approximation will be obtained by keeping only the first term in the series:

$$
\bar{K}_{W} \equiv \bar{C} \exp \left(\frac{i}{h} \bar{S}\right) \bar{A}_{0}
$$

The kernel $\bar{K}$ satisfies the Schrōdinger equation

$$
\left(H\left(\hat{Q}_{t}, \hat{P}_{t}\right)+\frac{\hbar}{i} \frac{\partial}{\partial t}\right) \bar{K}=0
$$

where (henceforth the subscript $t$ is omitted)

$$
\begin{aligned}
& \hat{Q}_{i}=Q_{i}, \quad \hat{Q}_{j}=-\sigma(j) \frac{n}{i} \frac{\partial}{\partial P_{j}} \\
& \hat{P}_{i}=\frac{n}{i} \frac{\partial}{\partial Q_{i}}, \quad \hat{P}_{j}=P_{j}
\end{aligned}
$$

and goes to the appropriate Fourier transform of the delta function as $t \rightarrow 0$. These conditions determine, in principle, the functions $\dot{A}_{i}$.

By commuting Schrödinger's operator through $\exp \left(\frac{i}{\lambda} \tilde{S}\right)$ we get

$$
\left(\hat{n}+\frac{\hbar}{i} \frac{\partial}{\partial t}\right) \exp \left(\frac{i}{h} \bar{S}\right)=\exp \left(\frac{i}{h} \bar{S}\right)\left(\tilde{D}_{0}+n \bar{D}_{1}+n^{2} \bar{D}_{2}+\ldots\right)
$$

where $\bar{D}_{i}$ are differential operators independent of $\hbar$. It will be shown below that $\bar{D}_{0}$ coincides with the Hamilton-Jacobi equation for $\tilde{S}$ and therefore vanishes identically. Substitution of (2.34) and (2.38) into Schrödinger's equation yields the following tower of differential equations

$$
\begin{aligned}
& \tilde{D}_{1} \ddot{A}_{0}=0 \\
& \tilde{D}_{2} \dot{A}_{0}+\tilde{D}_{1} \bar{A}_{1}=0 \\
& \tilde{D}_{3} \dot{A}_{0}+\tilde{D}_{2} \bar{A}_{1}+\bar{D}_{1} \bar{A}_{2}=0
\end{aligned}
$$


For a sufficiently regular Hamiltonian, these can be solved iteratively with appropriate boundary conditions to give an expression for the kernel valid for small $t .^{1}$ Here we are interested only in the first of these equations which, as we shall see, is solved by

$$
\dot{A}_{0}=\sqrt{\tilde{D}}
$$

where $\bar{D}$ is the generalized Van Vleck superdeterminant (2.24). This result is proven by obtaining $\bar{D}_{0}$ and $\bar{D}_{1}$ for a general Hamiltonian. The notation is somewhat simpler when the Hamiltonian is given in coordinate representation, i.e., when we consider $K\left(Q_{t}, Q_{0} ; t\right)$ and here we shall restrict ourselves to this case. The proof for general $\tilde{K}$ is essentially the same.

Since many quantum Hamiltonians may be associated with a classical system, we must specify to which particular one eq. (2.40) applies. We shall assume that the classical Hamiltonian is an analytic (super) function of $Q_{k}$ and $P_{h}$ and is expanded as a power series in $P_{h}$ :

$$
X(Q, P)=\sum_{r} g_{r}(Q) f_{r}(P)
$$

where $f_{r}$ is a homogeneous polynomial of degree $r$ in the momenta. The associated quantum Hamiltonian is taken to be

$$
\hat{H}=\mu(\hat{Q}, \hat{P})=\frac{1}{2} \sum_{r}\left[g_{r}(\hat{Q}), f_{r}(\hat{P})\right]_{+}
$$

where the bracket is

$$
|\hat{A}, \hat{B}|_{+}=\dot{A} \dot{B}+\sigma(A, B) \dot{B} \hat{A}
$$

\footnotetext{
'For longer times it might be necissary to add contributions from various classical trajectories connecting the initial and final confgurations [24\}.
} 


$$
\sigma(A, B)= \begin{cases}-1 & \text { if } \hat{A} \text { and } \dot{B} \text { are fermionic (odd) operators } \\ +1 & \text { otherwise }\end{cases}
$$

To compute $D_{0}$ and $D_{1}$ we first expand

$$
\begin{aligned}
f_{r}\left(\frac{\hbar}{i} \frac{\partial}{\partial Q}\right) e^{\hbar s} \sqrt{D} & =e^{i s}\left\{f_{r}\left(\frac{\partial S}{\partial Q}\right) \sqrt{D}+\frac{\hbar}{i} \sigma(k) \sigma\left(k, f_{r}\right) \frac{\partial f_{r}}{\partial P_{k}}\left(\frac{\partial S}{\partial Q}\right) \frac{\partial \sqrt{D}}{\partial Q_{k}}\right. \\
& \left.+\frac{\hbar}{i} \frac{1}{2} \frac{\partial}{\partial Q_{k}}\left(\frac{\partial f_{r}}{\partial P_{k}}\left(\frac{\partial S}{\partial Q}\right)\right) \sqrt{D}+0\left(\hbar^{2}\right)\right\}
\end{aligned}
$$

and

$$
\frac{n}{i} \frac{\partial}{\partial t} e^{i s} \sqrt{D}=e^{i s}\left\{\frac{\partial S}{\partial t} \sqrt{D}+\frac{n}{i} \frac{\partial \sqrt{D}}{\partial t}\right\}
$$

where $\sigma(k)$ has been defined in eq. (2.8).

Next, we observe that the bracket in eq. $(2.42)$ can be written

$$
\frac{1}{2}\left[g_{r}(\hat{Q}), f_{r}(\hat{P})\right]_{+}=g_{r}(\hat{Q}) f_{r}(\hat{P})+\frac{1}{2} \frac{\hbar}{i} \sigma\left(k, f_{r}\right) \frac{\partial g_{r}}{\partial Q_{k}}(\hat{Q}) \frac{\partial f_{r}}{\partial P_{k}}(\hat{P})+O\left(\hbar^{2}\right)
$$

Collecting the terms of order $\hbar^{0}$ from (2.45) and (2.46) with (2.44) yields

$$
D_{0}=\sum_{r} g_{r}(Q) f_{r}\left(\frac{\partial S}{\partial Q}\right)+\frac{\partial S}{\partial t}=\mathcal{N}\left(Q, \frac{\partial S}{\partial Q}\right)+\frac{\partial S}{\partial t}=0
$$

which, as mentioned above, is the Hamilton-Jacobi equation.

After multiplying by $\sqrt{D}$, the terms of order $\hbar$ are proportional to

$$
\begin{aligned}
& \frac{\partial D}{\partial t}+\sigma(k) \sigma\left(k, f_{r}\right) g_{r}(Q) \frac{\partial f}{\partial P_{k}}\left(\frac{\partial S}{\partial Q}\right) \frac{\partial D}{\partial Q_{k}}+g_{r}(Q) \frac{\partial}{\partial Q_{k}}\left(\frac{\partial f_{r}}{\partial P_{k}}\left(\frac{\partial S}{\partial Q}\right)\right) D \\
& +\sigma\left(k, f_{r}\right) \frac{\partial g_{r}}{\partial Q_{k}} \frac{\partial f_{r}}{\partial P_{k}}\left(\frac{\partial S}{\partial Q}\right) D=\frac{\partial}{\partial Q_{k}}\left(\frac{\partial}{\partial P_{k}}\left(g_{r}(Q) f_{r}\left(\frac{\partial S}{\partial Q}\right)\right) D\right)+\frac{\partial D}{\partial t}
\end{aligned}
$$

Summing over $r$ gives

$$
D_{1} \sqrt{D} \sim \frac{\partial}{\partial Q_{k}}\left(\frac{\partial N}{\partial P_{k}}\left(\frac{\partial S}{\partial Q}\right) D\right)+\frac{\partial D}{\partial t}=0
$$


This is the continuity equation satisfied by the superdeterminant $D$, and this part of the proof is thus completed. Had we considered $\bar{K}$ and $\tilde{D}$, we would have obtained eqs. (2.23) and (2.25) instead of (2.47) and (2.49), as is clear from eq. (2.37).

To complete the proof we still have to show that the WKB expression

$$
\tilde{K}_{W}=\bar{C} \exp \left(\frac{i}{h} \bar{s}\right) \sqrt{\bar{D}}
$$

has the correct limit as $t \rightarrow 0$. This will be done for the kernel $K_{W}^{\prime}\left(x_{t}, \bar{\theta}_{t}, x_{0}, \theta_{0} ; t\right)$.

The reason is that, although ultimately we want the kernel $K$ in coordinate representation (in order to compute traces, for instance), the corresponding action $S\left(x_{t}, \theta_{t}, x_{0}, \theta_{0} ; t\right)$ does not exist in general, as mentioned in section 1 . Once $K^{\prime}$ is known we can obtain $K$ by a fermionic Fourier transform.

As this part of the proof involves the study of the action when $t \rightarrow 0$, we shall have to make some additional assumptions about the system. We shall assume that the Lagrangian is of the following form

$$
\begin{aligned}
\mathcal{L} & =\frac{1}{2} g_{\mu \nu}(x) \dot{x}^{\mu} \dot{x}^{\nu}+i \bar{\theta}_{a} \dot{\theta}_{a}+\dot{x}^{\mu} B_{\mu}(x, \theta, \bar{\theta})-W(x, \theta, \bar{\theta}) \\
\mu, \nu & : 1 \ldots n \\
\text { a } & : 1 \ldots m
\end{aligned}
$$

and that $g_{\mu \nu}, B_{\mu}$ and $W$ are analytic, with $\operatorname{det}\left\|g_{\mu \nu}\right\| \equiv g \neq 0$. The canonical momenta are

$$
p_{\mu}=\frac{\partial \mathcal{L}}{\partial \dot{x}^{\mu}}=g_{\mu \nu} \dot{x}^{\nu}+B_{\mu} \quad, \quad \pi_{a}=\frac{\partial \mathcal{L}}{\partial \dot{\theta}_{a}}=-i \dot{\theta}_{a}
$$

and the Hamiltonian is given by

$$
H=\dot{x}^{\mu} p_{\mu}+\dot{\theta}_{a} \pi_{a}-\mathcal{L}=\frac{1}{2}\left(p_{\mu}-B_{\mu}\right) g^{\mu \nu}\left(p_{\nu}-B_{\nu}\right)+W
$$


It is easy to show (see Appendix E) that for this system $S^{\prime}$ has the following asymptotic form

$$
\begin{aligned}
S^{\prime}\left(x_{t}, \bar{\theta}_{t}, x_{0}, \theta_{0} ; t\right) & =\frac{1}{2 t}\left(g_{\mu \nu}\left(x_{0}\right) X^{\mu} X^{\nu}+O\left(X^{3}\right)\right)-i \bar{\theta}_{t a} \theta_{0 a}+O(X)+0(t) \\
X^{\mu} & \equiv x_{t}^{\mu}-x_{0}^{\mu}
\end{aligned}
$$

From this we obtain for the superdeterminant

$$
\sqrt{D^{\prime}}=\frac{1}{t^{n / 2}}\left(\sqrt{g\left(x_{0}\right)}+O(X)+0(t)\right)
$$

and therefore

$$
\lim _{t \rightarrow 0} \sqrt{D^{\prime}} \exp \left(\frac{i S^{\prime}}{\hbar}\right)=(2 \pi i \hbar)^{n / 2} \delta\left(x_{t}-x_{0}\right) \exp \left(\frac{1}{h} \bar{\theta}_{t a} \theta_{0 a}\right)
$$

This is, up to a constant, the Fourier transform with respect to $\theta_{t}$ of the delta function $\delta\left(x_{t}-x_{0}\right) \delta\left(\theta_{t}-\theta_{0}\right)$. This shows that $K_{W}^{\prime}$ has the correct limit as $t \rightarrow 0$. Using the conventions for the fermionic Fourier transform (see Appendix D) the constant of proportionality $C^{\prime}$ is determined and we have

$$
\begin{aligned}
K_{w}^{\prime}\left(x_{t}, \bar{\theta}_{t}, x_{0}, \theta_{0} ; t\right) & =C^{\prime} \exp \left(\frac{i}{\hbar} S^{\prime}\right) \sqrt{D^{\prime}} \\
C^{\prime} & =\frac{(-\hbar)^{m}}{(2 \pi i \hbar)^{n / 2}}
\end{aligned}
$$

where $n(m)$ is the number of bosonic (fermionic) coordinates $x^{\mu}\left(\theta_{a}\right)$, and $S^{\prime}$ and $D^{\prime}$ have been defined in eq. (2.26).

Before closing this section we discuss how to use this WKB formula with a quantum Hamiltonian $\mathcal{H}(\hat{Q}, \hat{P})$ which is not of the form given in eq. (2.42). For any $\hat{H}$ analytic in $\hat{Q}$ and $\hat{P}$, we can always commute the operators in such a way that we obtain a Hamiltonian of the form (2.42) plus terms 
proportional to $\hbar, \hbar^{2}$, etc.

$$
\begin{aligned}
& \hat{H}=H(\hat{Q}, \hat{P}) \equiv \frac{1}{2} \sum_{r}\left[g_{r}(\hat{Q}), f_{r}(\hat{P})\right]_{+}+\hbar \hat{\Theta}_{1}+\hbar^{2} \hat{\Theta}_{2}+\ldots \\
& \hat{\Theta}_{i}=\Theta_{i}(\hat{Q}, \hat{P})
\end{aligned}
$$

It is easy to see that, for purely bosonic hermitian Hamiltonians, $\Theta_{1}$ always vanishes. For instance, in coordinate representation

$$
\hat{p}_{\mu} g^{\mu \nu}(x) \hat{p}_{\nu}=\frac{1}{2}\left(\hat{p}_{\mu} \hat{p}_{\nu} g^{\mu \nu}+g^{\mu \nu} \hat{p}_{\mu} \hat{p}_{\nu}\right)+\hbar^{2} \partial_{\mu} \partial_{\nu} g^{\mu \nu}
$$

Since $\theta_{2}, \theta_{3} \ldots$ do not contribute to $\tilde{D}_{0}$ or $\tilde{D}_{1}$ (see eq. (2.38)), it is clear that the WKB formula can be used directly for a purely bosonic system, i.e., there are no ordering problems. This is not the case, however, for systems with fermionic variables. The following Hamiltonian

$$
\hat{\boldsymbol{\lambda}}=\hat{\boldsymbol{\theta}} \hat{\theta}
$$

is obviously hermitian, but is not of the form (2.42). To use the WKB formula, we first rewrite $\hat{x}$ as in (2.58)

$$
\hat{H}=\hat{\theta} \hat{\theta} \equiv \frac{1}{2}(\hat{\theta} \hat{\theta}-\hat{\theta} \hat{\theta})+\frac{\hbar}{2}, \quad \hat{\theta}_{1}=\frac{1}{2}
$$

and then consider a classical system with Hamiltonian

$$
x_{0}=\bar{\theta} \theta+\frac{a}{2}
$$

After obtaining $S^{\prime}$ and $D^{\prime}$ for this system, we recover the WKB approximation for the original system (2.60) by setting $a=\hbar$ and expanding $S^{\prime}$ to order $h$ and $S^{\prime}$ to order $\hbar^{0}$. The general rule is that one should always use as classical Hamiltonian

$$
H_{w}=H+\hbar \Theta_{1}
$$

rather than $H$. 


\subsection{WKB calculation of traces}

To obtain the trace of the evolution operator of a system with bosonic and fermionic variables in the WKB approximation we first compute $K_{W}^{\prime}\left(I_{l}, \bar{\theta}_{l}, I_{0}, \theta_{0} ; t\right)$ as given by eq. (2.57). A fermionic Fourier transform with respect to $\bar{\theta}_{t}$ gives $K_{W}$ in coordinate representation, and the trace is obtained by setting $x_{t}=x_{0}$ and $\theta_{t}=-\theta_{0}$ and integrating over initial coordinates

$$
\begin{gathered}
\left(\operatorname{tr} \exp \left(-\frac{i t}{\hbar} \hat{H}\right)\right)_{W}=\int d x_{0} \int_{\theta_{0}} K_{W}\left(x_{0},-\theta_{0}, x_{0}, \theta_{0} ; t\right) \\
=\frac{\hbar^{m}}{(2 \pi i \hbar)^{n / 2}} \int d x_{0} \int_{0_{0} \bar{\pi}_{0}} \sqrt{D^{\prime}\left(x_{0}, \bar{\theta}_{t}, x_{0}, \theta_{0} ; t\right)} \exp \frac{i}{h}\left(S^{\prime}\left(x_{0}, \bar{\theta}_{t}, x_{0}, \theta_{0} ; t\right)-i \bar{\theta}_{t} \theta_{0}\right)
\end{gathered}
$$

(See Appendix $\mathrm{D}$ for details on the derivation of this formula and eq. (2.67) below.)

The following trace can also be defined

$$
\operatorname{tr}(-)^{*} \exp \left(-\frac{i t}{\hbar} \hat{k}\right)
$$

where $\hat{F}$ is the fermion number operator

$$
\hat{\boldsymbol{F}}=\dot{\theta}_{a} \hat{\theta}_{\mathrm{d}}
$$

This trace is, in fact, the supertrace of the evolution operator, and in the WKB approximation is given by

$$
\begin{gathered}
\left(\operatorname{tr}(-)^{f} \exp \left(-\frac{i t}{\hbar} \hat{H}\right)\right)_{W} \\
\equiv\left(\operatorname{Str} \exp \left(-\frac{i t}{\hbar} \hat{H}\right)\right)_{W}=(-)^{m} \int d x_{0} \int_{0_{0}} K_{W}\left(x_{0}, \theta_{0}, x_{0}, \theta_{0} ; t\right) \\
=\frac{(-\hbar)^{m}}{(2 \pi i \hbar)^{n / 2}} \int d x_{0} \int_{D_{0} \bar{x}_{1}} \sqrt{D^{\prime}\left(x_{0}, \bar{\theta}_{l}, x_{0}, \theta_{0} i t\right)} \exp \frac{i}{\hbar}\left(S^{\prime}\left(x_{0}, \bar{\theta}_{t}, x_{0}, \theta_{0} ; t\right)+i \bar{\theta}_{t}, \theta_{0}\right)
\end{gathered}
$$


The WKB expressions for the kernel $K_{W}^{\prime}$, eq. (2.57), and for the trace and supertrace of the evolution operator, eq. (2.64) and (2.67), are important. As we shall see in chapter 3, the proof of the Atiyah-Singer index theorem reduces to the computation of eq. (2.67) for the appropriate supersymmetrical system.

We now consider the validity of the WKB expressions for the traces. In the case of sufficiently regular bosonic Hamiltonians $[23,24]$ it is well known that

$$
\lim _{t \rightarrow 0} \frac{K-K_{W}}{t}=0
$$

It is not too difficult to prove that eq. (2.68) holds for our more general system, eq. (2.51). But, because the kernel is singular at $t \rightarrow 0$, we cannot permute the order in which limits and traces are taken, and therefore eq. (2.68) does not imply

$$
\lim _{t \rightarrow 0} \frac{\operatorname{tr} K-\operatorname{tr} K_{W}}{t}=0
$$

Instead, as $t$ goes to zero we shall have

$$
\operatorname{tr} K-\operatorname{tr} K_{W} \sim 0\left(t^{\nu}\right)
$$

where the exponent $\nu$ will depend on the particular system considered.

We can obtain $\nu$ by studying the form of the quantum corrections $A_{\mathrm{i}}^{\prime}$ to the kernel $K_{W}^{\prime}$ (see eq. (2.34)). In order to keep the correct limit of the kernel as $t \rightarrow 0$, eq. (2.56), we must have

$$
\lim _{i \rightarrow 0} t^{n / 2} A_{i}^{\prime}\left(x_{i}=x_{0}\right)=0, \text { for } i>0
$$


Defining $^{2}$

$$
A^{\prime}=A_{0}^{\prime}+\hbar A_{1}^{\prime}+\hbar^{2} A_{2}^{\prime}+\ldots, A_{0}^{\prime}=\sqrt{D^{\prime}}
$$

by eqs.(2.55) and (2.71) we can write

$$
\begin{gathered}
A^{\prime}\left(x_{t}=x_{0}\right)=\frac{\sqrt{g\left(x_{0}\right)}}{t^{r / 2}}\left(1+b_{1} t+b_{2} t^{2} \ldots\right) \\
b_{i}=b_{i}\left(x_{0}, \bar{\theta}_{i}, \theta_{0}, \hbar\right)
\end{gathered}
$$

According to eq. (2.54) the modified classical action $S^{\prime}$ does not contain negative powers of $t$ for $x_{i}=x_{0}$, and we can write

$$
\begin{gathered}
S^{\prime}\left(x_{t}=x_{0}\right)=S_{0}^{\prime}+S_{1}^{\prime} t+S_{2}^{\prime} t^{2}+\ldots \\
S_{i}^{\prime}=S_{i}^{\prime}\left(x_{0}, \bar{\theta}_{i}, \theta_{0}, n\right)
\end{gathered}
$$

(Recall that, by our discussion at the end of section $2, S^{\prime}$ can contain terms linear in $\hbar$.

Combining eqs. (2.73) and (2.74) we obtain for the integrand in either (2.64) or (2.67)

$$
\text { Integrand } \begin{aligned}
& \sim \frac{\sqrt{g\left(x_{0}\right)}}{t^{n / 2}}\left(a_{0}+a_{1} t+a_{2} t^{2}+a_{3} t^{3}+\ldots\right) \\
a_{i} & =a_{i}\left(x_{0}, \bar{\theta}_{t}, \theta_{0}, n\right)
\end{aligned}
$$

Note that, for a purely bosonic system, the functions $a_{i}$ are the same as in the DeWitt-WKB expansion of the kernel [29].

By construction, the functions $b_{i}$ in eq. (2.73) will contain terms of order $n^{0}, \hbar^{1}, n^{2}$, etc. Only the terms of order $\hbar^{0}$ are included in the WKB approximation. From dimensional analysis and symmetry considerations it is ${ }^{2}$ If $D_{2}^{\prime} \sqrt{D^{\prime}}$ is equal to zero, the solution to (2.39) with the boundary conditions (2.71) is $A_{i}^{\prime}=0$ for $i>1$, and the WKB expression for the kernel is exact. This only happens for very particular simple oystems [23|. 
usually possible to determine the form of the terms depending on $\hbar$. If such a term occurs in $b_{i}$, it will give contributions to functions $a_{j}$ with $j \geq i$. Some of these contributions will vanish after we integrate, either because they appear as total derivatives or because the fermionic integrals are not saturated. Assume that the first non-vanishing contribution to the trace from a term depending on $\hbar$ in $A^{\prime}$ appears in $a_{k}$. Since such a contribution is necessarily missing in the WKB formula, we have as $t \rightarrow 0$

$$
\operatorname{tr} K-\operatorname{tr} K_{W} \sim O\left(t^{k-n / 2}\right)
$$

and we obtain $\nu=k-n / 2$. This is only a lower bound because the contribution could vanish accidentally.

As a simple example, we consider the following Hamiltonian

$$
\hat{H}=\frac{1}{2} \hat{p}_{\mu} \hat{p}_{\mu}+V(\hat{x}), \mu=1, \ldots, n
$$

where $V$ is a completely arbitrary analytic potential. The relevant dimensions are

$$
[\hbar] \sim \frac{x^{2}}{t} \quad|V| \sim \frac{x^{2}}{t^{2}}
$$

The following terms, invariant under rotations, can appear in $A\left(x_{t}=x_{0}=0\right)$

$\begin{array}{ccc} & \text { Dimension } & \text { Terms } \\ b_{1} & t^{-1} & - \\ b_{2} & t^{-2} & V_{\mu \mu} \\ b_{3} & t^{-s} & h V_{\mu \mu \nu \nu}\end{array}$

where $V_{\mu \mu}=\partial_{\mu} \partial_{\mu} V\left(x=x_{0}\right)$, etc. 
By time reversal invariance the action contains only odd powers of $t$ :

$$
\begin{array}{ccc} 
& \text { Dimension } & \text { Terms } \\
S_{1} & x^{2} / t^{2} & V \\
S_{3} & x^{2} / t^{4} & V_{\mu} V_{\mu}
\end{array}
$$

The term $h V_{\mu \mu \nu \nu}$, missing in the WKB formula, gives the following contributions $\Delta a_{i}$ to the integrand

$$
\begin{aligned}
\text { Integrand } & =\exp \left(\frac{i}{h}\left(S_{1} t+S_{3} t^{3}+\ldots\right)\right) \frac{1}{t^{n / 2}}\left(1+b_{1} t+b_{2} t^{2}+\ldots\right) \\
& =\frac{1}{t^{n / 2}}\left(a_{0}+a_{1} t+a_{2} t^{2}+\ldots\right) \\
\Delta a_{0}=\Delta a_{1} & =\Delta a_{3}=0, \Delta a_{3} \sim n V_{\mu \mu \nu \nu}, \Delta a_{4} \sim V V_{\mu \mu \nu \nu}+\ldots
\end{aligned}
$$

Since $\Delta a_{3}$ is a total derivative, by eq. (2.76) we get $\nu=4-n / 2$ and

$$
\lim _{t \rightarrow 0}\left(\operatorname{tr} K-\operatorname{tr} K_{W}\right) \begin{cases}=0, & n<8 \\ =\infty, & n>8\end{cases}
$$

Below eight dimensions the WKB trace is exact in the limit $t \rightarrow 0$. Note that the naive expectation, eq. (2.69), fails for $n \geq 6$. 


\section{Added Note}

The classical mechanics of Bose-Fermi systems is treated in detail in refs. $\quad[30-32]$, and the corresponding quantum description can be found in ref. [33]. This last reference contains also a presentation of the WKB approximation for purely fermionic systems, but the Bose-Fermi case, relevant to our computation of the index, is not considered.

The concept of Weyl symbol of a fermionic operator is studied in ref. [34]. This is relevant to the present work in that the Hamiltonian $H_{w}$ of eq. (2.63) is, to order $\hbar$, the Weyl symbol of the operator $\hat{k}$. 


\section{WKB PROOF OF THE INDEX THEOREM}

A new proof of the Atiyah-Singer index theorem has been given independently by Alvarez-Gaumé [13] and by Friedan and Windey $[14,15]$, who have followed Witten's suggestion that the index formula could be understood in terms of a suitable quantum mechanical supersymmetric system. These authors have used path integral techniques to compute the index, which they have expressed as the supertrace of the evolution operator for the system. Rigorous mathematical derivations along similar lines have been subsequently given by Getzler [20] (who uses a kind of Hamiltonian description) and by Bismut [21] (who uses Wiener integrals to give rigorous estimates).

In this chapter we follow the approach in Refs. [13-15] but, instead of a path integral, we use a WKB expression to evaluate the index [16]. This WKB expression is given in terms of classical quantities, namely a modified form of the classical Hamilton-Jacobi function and a generalization of the Van Vleck determinant [22]. In this way the evaluation of the index formula is reduced to a problem in supersymmetric classical mechanics.

We describe the calculation of the index for the case of the Dirac operator on a compact Riemannian manifold of even dimension $n$ in the presence of an external Yang-Mills potential. This twisted spin complex is known to contain all classical geometric complexes: de Rham (Euler), signature (Hirzebruch), Dolbeault [17). The index theorem for the twisted Dirac operator implies that for 1 general elliptic complex, in the sense that there is a homotopy 
relation.

The Dirac operator in the presence of an external Yang-Mills field $A_{\mu}$ is given by

$$
D=\gamma^{a} e_{a}^{\mu}(x) D_{\mu}
$$

where $e_{a}^{\mu}$ is the inverse vielbein field and the $x$-independent $\gamma$ matrices satisfy

$$
\left\{\gamma^{a}, \gamma^{b}\right\}=2 \delta^{a b}, \gamma^{a t}=\gamma^{a} ; a, b: 1 \ldots n
$$

$D_{\mu}$ is covariant with respect to spinor and internal indices [17]

$$
\begin{aligned}
D_{\mu} & =\partial_{\mu}+\frac{1}{8} \omega_{\mu a b}\left[\gamma^{a}, \gamma^{\alpha}\right]+A_{\mu} \\
\left(A_{\mu}^{i k}\right)^{*} & =-A_{\mu}^{k i} ; i, k: 1 \ldots p
\end{aligned}
$$

The index of the Dirac operator is the difference between the number $N_{+}$of zero modes of positive chirality and the number $N_{-}$of zero modes of negative chirality. There is a simple formula for the index

$$
\begin{aligned}
N_{+}-N_{-} & =\operatorname{tr}\left(\gamma_{5} \exp \left(\beta D^{\dagger} D\right)\right) \\
\gamma_{5} & =\gamma^{1} \gamma^{2} \ldots \gamma^{n}
\end{aligned}
$$

and the result is independent of $\beta$. This formula has been discussed in several recent papers $[13-15,16]$, to which we refer the reader for a detailed explanation. Here we shall concern ourselves with the use of the formalism developed in previous sections to compute (3.4).

From eqs. (3.1) and (3.3) $D^{\dagger} D$ is

$$
D^{\dagger} D=D D^{\dagger}=\frac{1}{\sqrt{g}} D_{\mu} \sqrt{g} g^{a \nu} D_{\nu}+\frac{1}{4} F_{a b}\left|\gamma^{a}, \gamma^{b}\right|-\frac{1}{4} R
$$


where $R$ is the scalar curvature and

$$
F_{a b}=e_{a}^{\mu} e_{b}^{\nu} F_{\mu \nu}=e_{a}^{\mu} e_{b}^{\nu}\left(\partial_{\mu} A_{\nu}-\partial_{\nu} A_{\mu}+\left|A_{\mu}, A_{\nu}\right|\right)
$$

in matrix notation.

The operator $D^{\dagger} D$ is hermitian if the measure is taken to be $\sqrt{g} d x$, i.e.,

$$
\operatorname{tr} \int d x \sqrt{g} \Psi_{1}^{\dagger}\left(D^{\dagger} D \Psi_{2}\right)=\operatorname{tr} \int d x \sqrt{g}\left(D^{\dagger} D \Psi_{1}\right)^{\dagger} \Psi_{2}
$$

where $\Psi_{1}$ and $\Psi_{2}$ are arbitrary spinors and the trace is over spinor and internal indices. The operator

$$
g^{1 / 4} D^{\dagger} D g^{-1 / 4}
$$

is hermitian with measure $d x$ and has the same spectrum as $D^{\dagger} D$. In the next section we shall write (3.8) as a quantum mechanical (supersymmetric) Hamiltonian to which the formalism developed in chapter 2 can be applied. Section 3 is dedicated to the study of the validity of the WKB formula for the index, which is actually evaluated in section 4.

\subsection{WKB formula for the index}

We may write eq. (3.8) as a quantum mechanical Hamiltonian by identifying

$$
\hat{p}_{\mu}=\frac{n}{i} \partial_{\mu}
$$

and expressing the matrices in terms of fermionic operators in the usual way $\{15,31,32\}$

$$
\hat{\psi}^{a}=\frac{\hbar^{1 / 2}}{\sqrt{2}} \gamma^{a} \quad \hat{\bar{\eta}}_{i} A_{\mu}^{i k} \hat{\eta}_{k}=\hbar A_{\mu}
$$


44

$$
\begin{gathered}
\left\{\hat{\psi}^{a}, \hat{\psi}^{b}\right\}=\hbar \delta^{a b} \quad\left\{\hat{\bar{\eta}}_{i}, \hat{\eta}_{k}\right\}=\hbar \delta_{i k} \\
\hat{\psi}^{a \dagger}=\hat{\psi}^{a}, \hat{\eta}_{k}^{\dagger}=\hat{\bar{\eta}}_{k} \\
a, b: 1, \ldots, n \quad i, k: 1, \ldots, p
\end{gathered}
$$

The result is

$$
\begin{aligned}
\hat{H} \equiv-\frac{\hbar^{2}}{2} g^{1 / 4} D^{\dagger} D g^{-1 / 4} & =\frac{1}{2} \hat{D}_{\mu} g^{\mu \nu} \hat{D}_{\nu}-\frac{1}{4} \hat{\bar{\eta}}_{i} F_{a b}^{i k} \hat{\eta}_{k}\left[\hat{\psi}^{a}, \hat{\psi}^{b}\right] \\
& +\frac{1}{4} \hbar^{2} R+\frac{1}{2} \hbar^{2} g^{-1 / 4} \partial_{\mu}\left(g^{\mu \nu} \partial_{\nu} \sqrt{g}\right)
\end{aligned}
$$

where

$$
\hat{D}_{\mu} \equiv \hat{p}_{\mu}-\frac{1}{4}\left[\hat{\psi}^{a}, \hat{\psi}^{b}\right] \omega_{\mu a b}-i \hat{\eta}_{i} A_{\mu}^{i h} \hat{\eta}_{k}
$$

The hermitian operators $\hat{\psi}^{\star}$ may be related to creation and annihilation operators $\hat{\xi}_{p}, \hat{\xi}_{p}$ by

$$
\begin{aligned}
& \hat{\xi}_{r} \equiv \frac{1}{\sqrt{2}}\left(\hat{\psi}^{2 r-1}+i \hat{\psi}^{2 r}\right), \hat{\bar{\xi}}_{r}=\hat{\xi}_{r}^{\uparrow},\left\{\hat{\bar{\xi}}_{r}, \hat{\xi}_{s}\right\}=n \delta_{r,} \\
& r, s: 1 \ldots n / 2
\end{aligned}
$$

The matrix $\gamma_{s}=\gamma^{1} \gamma^{2} \ldots \gamma^{n}$ anticommutes with all the creation operators $\bar{\xi}_{r}$ and if we define the vacuum $|0\rangle$ so that

$$
\begin{aligned}
& \hat{\xi}_{r}|0\rangle=0, \quad \forall r: 1 \ldots n / 2 \\
& \gamma_{s}|0\rangle=+|0\rangle
\end{aligned}
$$

then we can identify

$$
\gamma_{s} \equiv(-)^{\hat{F}_{t}}, \hat{F}_{\epsilon}=\dot{\bar{\xi}}_{r} \dot{\xi}_{r}
$$

The "total" fermion number operator $\bar{F}$ is defined by

$$
\hat{F}=\hat{F}_{\xi}+\hat{F}_{\eta}=\hat{\bar{\xi}}_{r} \dot{\xi}_{r}+\hat{\bar{\eta}}_{i} \hat{\eta}_{i}
$$


If we now compute

$$
I(\alpha) \equiv \operatorname{tr}(-)^{F} \exp \left(\frac{-i t}{\hbar}\left(\hat{k}-\frac{\alpha}{t} \hat{F}_{\eta}\right)\right)
$$

and expand

$$
I(\alpha)=\sum_{\ell=0}^{p}(-)^{\ell} e^{i \alpha \ell} I_{\ell}
$$

then $I_{1}$ coincides with the index $N_{+}-N_{-}$as given by eq. (3.4). The introduction of the chemical potential $\alpha[15]$ permits the separation of the contributions to the trace (3.17) from the different antisymmetric powers $T^{\wedge \ell}, T$ being the representation of the fermions in the Dirac equation.

The Hamiltonian $\hat{k}-\alpha / t \hat{F}_{\eta}$ is not in the form of eq. (2.42). Following the discussion at the end of Section 3, we simply make the substitution

$$
\hat{\bar{\eta}}_{i} \hat{\eta}_{k} \rightarrow \frac{1}{2}\left(\hat{\bar{\eta}}_{i} \hat{\eta}_{k}-\hat{\eta}_{k} \hat{\bar{\eta}}_{i}\right)+\frac{1}{2} \hbar
$$

The classical Hamiltonian $H_{W}$ to be used in the computation of $S^{\prime}$ is obtained by neglecting terms on order $\hbar^{2}$. The result is

$$
K_{W}=\frac{1}{2} D_{\mu} g^{\mu \nu} D_{\nu}-\frac{1}{2} \bar{\eta}_{i} F_{a b}^{i k} \eta_{L} \psi^{e} \psi^{b}-\frac{\alpha}{t} \bar{\eta}_{i} \eta_{i}-\frac{\hbar}{4} F_{a b}^{i i} \psi^{\alpha} \psi^{b}-\frac{\hbar}{2} \frac{p \alpha}{t}
$$

where ${ }^{1}$

$$
D_{\mu}=p_{\mu}-\frac{i}{2} \omega_{\mu a b} \psi^{a} \psi^{b}-i \bar{\eta}_{i} A_{\mu}^{i h} \eta_{h}-\frac{i}{2} \hbar A_{\mu}^{i i}
$$

By using

$$
\dot{x}_{\mu}=\frac{\partial X_{W}}{\partial p_{\mu}}=g^{\mu \nu} D_{\nu}
$$

\footnotetext{
${ }^{1}$ Note that this Hamiltonian is of the type (2.53) and we can use the WKB expressions previously derived.
} 
we can construct the Lagrangian $L_{W}$

$$
\begin{aligned}
\mathcal{L}_{w} & \equiv \dot{x}^{\mu} p_{\mu}+i \bar{\xi}_{e} \dot{\xi}_{r}+i \bar{\theta}_{i} \dot{\theta}_{i}-\not_{w} \\
& =\frac{1}{2} g_{\mu \nu} \dot{x}^{\mu} \dot{x}^{\nu}+\frac{i}{2} \psi^{a} D_{t} \psi^{a}+i \bar{\eta}_{i} D_{t} \eta_{i}+\frac{\alpha}{t} \bar{\eta}_{i} \eta_{i}=\ldots \\
& +\frac{1}{2} \bar{\eta}_{i} F_{a b}^{i k} \eta_{k} \psi^{a} \psi^{b}+\frac{i \hbar}{2} \dot{x}^{\mu} A_{\mu}^{i i}+\frac{\hbar \alpha p}{2 t} \\
& +\frac{1}{4} \hbar F_{a b}^{i j} \psi^{a} \psi^{b}-\frac{1}{4} \frac{d}{d t}\left(\psi^{a} \Omega_{a b} \psi^{b}\right)
\end{aligned}
$$

where the covariant time derivatives are given by

$$
\begin{aligned}
D_{s} \psi^{a} & \equiv \dot{\psi}^{a}+\dot{x}^{\mu} \omega_{\mu a b} \psi^{b} \\
D_{i} \eta_{i} & \equiv \dot{\eta}_{i}+\dot{x}^{\mu} A_{\mu i k} \eta_{k}
\end{aligned}
$$

The total derivative term comes from the identity

$$
\begin{gathered}
\bar{\xi}_{r} \dot{\xi}_{r}=\frac{1}{2} \psi^{a} \dot{\psi}^{a}+\frac{i}{2} \psi^{a} \Omega_{a b} \dot{\psi}^{b}=\frac{1}{2} \psi^{a} \dot{\psi}^{a}+\frac{i}{4} \frac{d}{d t}\left(\psi^{a} \Omega_{a b} \psi^{b}\right) \\
r: 1, \ldots, n / 2 \quad a, b: 1, \ldots, n \\
\Omega:\left[\begin{array}{rrrr}
0 & 1 & 0 \\
-1 & 0 & & \\
0 & 0 & 1 \\
& -1 & 0
\end{array}\right]
\end{gathered}
$$

Although this total derivative does not affect the equations of motion, it cannot be neglected in the computation of the classical action, because $\psi^{\circ} \Omega_{a b} \psi^{b}$ is not a conserved quantity.

Defining a supersymmetry variation by

$$
\begin{array}{ll}
\mathbf{S} x^{\mu}=\psi^{\mu} & \mathbf{S} f_{i}=-\dot{\eta}_{i} \quad \mathbf{S}^{2}=i \frac{d}{d t} \\
\mathbf{S} \psi^{\mu}=i \dot{x}^{\mu} & \mathbf{S} \eta_{i}=-i f_{i}
\end{array}
$$




$$
\psi^{\mu}=e_{a}^{\mu}(x) \psi^{a}
$$

the Lagrangian $\mathcal{L}_{W}$ can be expressed as

$$
\mathcal{L}_{w}=\mathrm{S} X-\frac{1}{4} \frac{d}{d t}\left(\psi^{a} \Pi_{a b} \psi^{b}\right)+\frac{\alpha}{t} \bar{\eta}_{i} \eta_{i}
$$

where the (odd) function $X$ is

$$
X=\frac{1}{2 i} g_{\mu \nu}(x) \dot{x}^{\mu} \psi^{\nu}+f_{i} \bar{\eta}_{i}+\psi^{\mu} \bar{\eta}_{i} A_{\mu}^{i k} \eta_{k}+\frac{\hbar}{2} \psi^{\mu} A_{\mu}^{i i}
$$

and the auxiliary fields $f_{i}, \bar{f}_{i}$, are eliminated by using the equations of motion

$$
f_{i}=-i \psi^{\mu} A_{\mu}^{i k} \eta_{k} \quad \bar{f}_{i}=i \psi^{\mu} \bar{\eta}_{k} A_{\mu}^{k i}
$$

Then we see that the variation of $\mathcal{L}_{W}$ is a total derivative, because

$$
s^{2} X=i \dot{X}, s\left(\bar{\eta}_{i} \eta_{i}\right)=0
$$

This shows explicitly that the system is supersymmetric.

We can obtain a WKB approximation for $I(\alpha)$ by writing eq. (2.67) with $(\xi, \eta)$ instead of $\theta$ and $m=n / 2+p$

$$
I(\alpha)_{W}=\lim _{i \rightarrow 0} C^{\prime} \int d x_{0} \int_{\xi_{0} \eta_{0}} \int_{\bar{\pi}_{i} \bar{\xi}_{1}} \sqrt{D^{\prime}(\cdot)} \exp \frac{i}{\hbar}\left(S(\cdot)-i \bar{\xi}_{t} \Delta \xi(\cdot)-i \bar{\eta}_{t} \Delta \eta(\cdot)\right)
$$

where

$$
\begin{gathered}
C^{\prime}=(-)^{n / 2} \frac{(-n)^{p}}{(2 \pi i)^{n}}, \Delta \xi(\cdot) \equiv \xi_{t}(\cdot)-\xi_{0}, \quad(\cdot) \equiv\left(x_{t}=x_{0}, \bar{\xi}_{t}, \bar{\eta}_{t}, x_{0}, \xi_{0}, \eta_{0} ; t\right) \\
\Delta \eta(\cdot) \equiv \eta_{t}(\cdot)-\eta_{0}
\end{gathered}
$$

and $S(\cdot)$ is given by the integral of $C_{w}$ along the classical trajectory specified by $(\cdot)$. 


\subsection{Validity of the WKB approximation}

To evaluate eq. (3.31) we need the equations of motion. By varying $\mathcal{L}_{W}$ we obtain

$$
\begin{gathered}
D_{b} \dot{x}^{\mu}=\left(\frac{i}{2} R^{\mu}{ }_{\nu a b} \psi^{a} \psi^{b}+i \bar{\eta}_{i} F^{i j \mu}{ }_{\nu} \eta_{j}+\frac{i}{2} \hbar F^{i i \mu}{ }_{\nu}\right) \dot{x}^{\nu}+\ldots \\
+\frac{1}{2} \bar{\eta}_{i}\left(D^{\mu} F\right)_{a b}^{i j} \eta_{j} \psi^{a} \psi^{b}+\frac{\hbar}{4}\left(D^{\mu} F\right)_{a b}^{i i} \psi^{a} \psi^{b} \\
D_{b} \psi^{a}=i \bar{\eta}_{i} F_{a b}^{i j} \eta_{j} \psi^{b}+\frac{i \hbar}{2} F_{a b}^{i i} \psi^{b} \\
D_{i} \eta_{i}=\frac{i}{2} F_{a b}^{i j} \psi^{a} \psi^{b} \eta_{j}+\frac{i \alpha}{t} \eta_{i}
\end{gathered}
$$

where

$$
\begin{aligned}
D_{t} \dot{x}^{\mu} & =\dot{x}^{\mu}+\Gamma_{a \beta}^{\mu} \dot{x}^{\alpha} \dot{x}^{\rho} \\
\left(D^{\mu} F\right)_{a b}^{i j} & =F_{a b}^{i j, \mu}-\left[F_{a b}, A^{\mu}\right]^{i j}-\left[F^{i j}, \omega^{\mu}\right]_{a b}
\end{aligned}
$$

Since the Hamiltonian $H_{W}$ is real, the equation of motion for $\bar{\eta}_{i}$ is the complex conjugate of $(3.33 \mathrm{c})$.

After we substitute eqs.(115b-c) into $\mathcal{L}_{w}$, the function $S(\cdot)$ can be written

$$
\begin{aligned}
S(\cdot) \equiv \int_{0}^{t} \mathcal{L}_{w} d t^{\prime} & =\int_{0}^{t} d t^{\prime}\left(\frac{1}{2} g_{\mu \nu} \dot{x}^{\mu} \dot{x}^{\nu}-\frac{1}{2} \bar{\eta}_{i} F_{a b}^{i j} \eta_{j} \psi^{a} \psi^{b}+\frac{i \hbar}{2} A_{\mu}^{i i} \dot{x}^{\mu}\right)+\frac{\hbar}{2} p \alpha \\
& -\frac{1}{4} \Delta\left(\psi^{a} \Omega_{a b} \psi^{b}\right)
\end{aligned}
$$

As explained in appendix $E$, the equations of motion can be solved iteratively and, for an analytic Hamiltonian (which we shall assume), the integrand in eq. (3.31) will be an analytic function of the variables $\left(x_{0}, \bar{\xi}_{t}, \xi_{0}, \bar{\eta}_{t}, \eta_{0}\right)$ and the fields and their derivatives evaluated at $x_{0}$. This fact allows the use of dimensional analysis to study the validity of the WKB approximation for 
$I(\alpha)$. The following dimensions are relevant

$$
\begin{aligned}
& |\hbar| \sim \mid \bar{\xi} \xi] \sim|\bar{\eta} \eta| \sim x^{2} / t \\
& {[g] \sim[\alpha] \sim 1} \\
& {[A] \sim[\omega] \sim 1 / x} \\
& {[R] \sim[F] \sim 1 / x^{2}}
\end{aligned}
$$

Note that only $h$ and the fermionic variables have non-vanishing (negative) time dimensions.

Because of the fermionic integration over $\bar{\xi}_{t}$ and $\xi_{0}$, the only terms in the integrand which can give a contribution to the trace must be proportional to

$$
\bar{\xi}_{t}^{1} \xi_{0}^{1} \ldots \bar{\xi}_{t}^{n / 2} \xi_{0}^{n / 2} \equiv\left(\bar{\xi}_{t} \xi_{0}\right)^{n / 2}
$$

By eq. (2.55) the square root of the superdeterminant for $x_{t}=x_{0}$ is of the following form

$$
\sqrt{D^{n}}=\frac{\sqrt{g\left(x_{0}\right)}}{t^{n / 2}}(1+0(t)) \equiv \frac{\sqrt{g\left(x_{0}\right)}}{t^{n / 2}} f_{0}(t), \quad\left[f_{0}(t)\right] \sim 1
$$

From the dimensions in eq. (3.31) it is clear that $f_{0}(t)$ will contain terms proportional to

$$
\xi t^{\prime}, s \geq \frac{r}{2}
$$

where $\xi$ stands for $\bar{\xi}_{1}$ and $\xi_{0}$.

If $\alpha, F_{a b}^{i j}\left(x_{0}\right)$ and $\left(D^{\mu} F\right)_{a b}^{i j}\left(x_{0}\right)$ are set equal to zero, the equations of motion have the following solution

$$
\begin{array}{rlrl}
x\left(t^{\prime}\right)=x_{0} & \eta\left(t^{\prime}\right)=\eta_{0} & \xi\left(t^{\prime}\right)=\xi_{0} \\
\bar{\eta}\left(t^{\prime}\right)=\bar{\eta}_{0} & \bar{\xi}\left(t^{\prime}\right)=\bar{\xi}_{0}
\end{array}
$$


and the exponent in eq. (3.31) vanishes. Since it also vanishes for $t \rightarrow 0$ with $x_{t}=x_{0}$, it has to be at least linear in some of the following monomials

$$
\bar{\eta}_{t} F\left(x_{0}\right) \eta_{0} t, \hbar F\left(x_{0}\right) t, \bar{\eta}_{t} D F\left(x_{0}\right) \eta_{0} t, \hbar D F\left(x_{0}\right) t, \hbar \alpha t
$$

As a consequence, the terms in the exponent will also be proportional to (3.39), and the only terms in the integrand which do not vanish upon integration over $\bar{\xi}_{t} \xi_{0}$ are proportional to

$$
\frac{1}{t^{n / 2}}\left(\bar{\xi}_{t} \xi_{0}\right)^{n / 2} t^{\bullet}=\left(\bar{\xi}_{t} \xi_{0}\right)^{n / 2} t^{0-n / 2}, s \geq n / 2
$$

In the limit $t \rightarrow 0$ only the term with $s=n / 2$ (i.e., $a_{n / 2}$ of eq. (3.75)) survives. This shows that the WKB expression approaches a constant value as $t$ goes to zero. ${ }^{2}$ To see whether this value is modified by corrections of higher order in $h$, we note that by eqs. (2.72) and (2.73) such corrections appear in the following form

$$
\begin{aligned}
\sqrt{D^{u}} \longrightarrow \sqrt{D^{\prime}}+h A_{1}^{\prime}+\hbar^{2} A_{2}^{\prime}+\ldots & =\frac{\sqrt{g\left(x_{0}\right)}}{t^{n / 2}}\left(f_{0}(t)+\hbar f_{1}(t)+\hbar^{2} f_{2}(t)+\ldots\right) \\
{\left[f_{i}(t)\right] } & \sim t^{i} / x^{2 i}
\end{aligned}
$$

and therefore $f_{i}(t)$ contributes terms proportional to

$$
\xi^{*} t^{*}, s \geq \frac{r}{2}+i
$$

These, after being multiplied by the exponential, will give contributions to the integrand proportional to

$$
\left(\bar{\xi}_{t} \xi_{0}\right)^{n / 2} t^{a-n / 2}, s \geq n / 2+i
$$

${ }^{2}$ Compare with the non-supersymmetric aystem considered in section 2.3 , for which the trace diverges as $t \rightarrow 0$. 
which vanish as $t \rightarrow 0$ for $i>0$. This shows that in the limit $t \rightarrow 0$ the WKB formula is exact, i.e.

$$
I(\alpha)_{w}=I(\alpha)
$$

(In the language of chapter 2 we would say that $\nu=1, \forall n, p$.)

Although $I(\alpha)$ is by construction independent of $t$ (eq. (3.4) is independent of $\beta$ ), the WKB approximation of eq. (3.31) will be, in general, time dependent. This dependence is cancelled by corrections of higher order in $\hbar$. The fact that such corrections are at least linear in $t$ justifies the calculation of $I(\alpha)$ as the limit $t \rightarrow 0$ of the WKB approximation.

\subsection{Computation of the index}

In order to evaluate eq. (3.31) we need to know the solution to the equations of motion (3.33) with the given boundary conditions through order $t^{n / 2}$. Since $n$ is arbitrary, it is obvious that we must simplify the equations before we attempt to solve them. There is a systematic way to do this without affecting the Enal result of the calculation. By eq. (3.42), the only terms which do not vanish upon integration over $\bar{\xi}_{t} \xi_{0}$ are proportional to

$$
\left(\bar{\xi}_{t} \xi_{0}\right)^{n / 2} t^{\ell}, \ell \geq 0
$$

Under a rescaling

$$
(\xi, \bar{\xi}) \longrightarrow\left(\beta^{-1 / 2} \xi, \beta^{-1 / 2} \bar{\xi}\right) \quad t \rightarrow \beta t
$$

they behave as $\beta^{\ell-n / 2}$. Only the terms of lowest order in $\beta$ (order $\beta^{-n / 2}$ ) survive in the limit $t \rightarrow 0$. Thus, it is enough to compute the solutions to 
the equations of motion to lowest order in $\beta$, which replaces $t$ as the natural parameter of the expansion.

Since all quantities in the Lagrangian have vanishing or negative time dimensions (see eq. (3.36)), any polynomial function of time dimension $r$ will be, at best, of order $\beta r$. By our discussion in appendix $E$, the solutions to the equations of motion will be (infinite) polynomials in $t, x_{0}, \bar{\xi}_{t}, \bar{\eta}_{l}, \xi_{0}, \eta_{0}$ and the fields and their derivatives at $x_{0}$.

We have already mentioned that if $\alpha, F\left(x_{0}\right)$ and $D^{\mu} F\left(x_{0}\right)$ are zero, a solution is given by $x\left(t^{\prime}\right)=x_{0}$. Therefore, $x\left(t^{\prime}\right)-x_{0}$ must be at least linear in some of the monomials in eq. (3.41), which have time dimension equal to zero and are of order $\beta$. Thus

$$
x\left(t^{\prime}\right)-x_{0} \sim o(\beta)
$$

By similar arguments it is easy to obtain

$$
\eta\left(t^{\prime}\right) \sim 0(1) \quad \psi\left(t^{\prime}\right)-\psi_{0} \sim 0\left(\beta^{1 / 2}\right) \quad \psi_{0} \sim 0\left(\beta^{-1 / 2}\right)
$$

and therefore

$$
D_{t} \dot{x} \sim 0\left(\beta^{-1}\right) \quad D_{t} \psi \sim 0\left(\beta^{-1 / 2}\right) \quad D_{1} \eta \sim 0\left(\beta^{-1}\right)
$$

The "effective equations of motion" are obtained by neglecting higher powers of $\beta$. Using a coordinate system and gauge such that

$$
A_{\mu}^{i j}\left(x_{0}\right)=w_{\mu b b}\left(x_{0}\right)=\partial_{\mu} g_{a \rho}\left(x_{0}\right)=\Gamma_{a \rho}^{\mu}\left(x_{0}\right)=0
$$

the result is

$$
\begin{aligned}
\ddot{x}^{\mu} & =\frac{i}{2} R_{\nu a b}^{\mu}\left(x_{0}\right) \psi_{0}^{a} \psi_{0}^{b} \dot{x}^{\nu}+\frac{1}{2} \bar{\eta}_{i} F_{a b}^{\prime j, \mu}\left(x_{0}\right) \psi_{0}^{a} \psi_{0}^{b} \eta_{j} \\
& +\frac{h}{4} F_{a b}^{i i, \mu}\left(x_{0}\right) \psi_{0}^{a} \psi_{0}^{b}+O(1)
\end{aligned}
$$




$$
\begin{gathered}
\dot{\psi}^{a}=i \bar{\eta}_{i} F_{a b}^{i j}\left(x_{0}\right) \eta_{j} \psi_{0}^{b}+\frac{i \hbar}{2} F_{a b}^{i i} \psi_{0}^{b}+0\left(\beta^{1 / 2}\right) \\
\dot{\eta}_{i}=\frac{i \alpha}{t} \eta_{i}+\frac{i}{2} F_{a b}^{i j}\left(x_{0}\right) \psi_{0}^{a} \psi_{0}^{b} \eta_{j}+O(1)
\end{gathered}
$$

To lowest order in $\beta$ the function $S$ as given by eq. (3.35) is

$$
\begin{aligned}
S= & -\frac{1}{2} \int_{0}^{t} \bar{\eta}_{i}\left(t^{\prime}\right) F_{a b}^{i j}\left(x_{0}\right) \psi_{0}^{a} \psi_{0}^{b} \eta_{j}\left(t^{\prime}\right) d t^{\prime}+\frac{\hbar}{2} p \alpha \\
& -\frac{1}{2} \psi_{0}^{a} \Omega_{a b} \Delta \psi^{b}+O(\beta)
\end{aligned}
$$

The second term in the exponent can be written

$$
\bar{\xi}_{t} \Delta \xi=\frac{1}{2} \psi_{i}^{a} \Delta \psi^{a}+\frac{i}{2} \psi_{i}^{a} \Omega_{a b} \Delta \psi^{b}
$$

which, by integrating eq. ( $3.53 \mathrm{~b})$ becomes

$$
\begin{aligned}
\bar{\xi}_{t} \Delta \xi & =\frac{i}{2} \psi_{0}^{a} \int_{0}^{t} \bar{\eta}_{i}\left(t^{\prime}\right) F_{a b}^{i j}\left(x_{0}\right) \eta_{j}\left(t^{\prime}\right) \psi_{0}^{b} d t^{\prime}+\frac{i h}{4} F_{a b}^{i i}\left(x_{0}\right) \psi_{0}^{a} \psi_{0}^{b} t \\
& +\frac{i}{2} \psi_{0}^{a} \Omega_{a b} \Delta \psi^{b}+O(\beta)
\end{aligned}
$$

Equation (3.53c) can be integrated to give, in matrix notation

$$
\eta_{1}=\exp (i \alpha+i \xi t) \eta_{0}+o(\beta)
$$

where we have defined

$$
F^{i j} \equiv \frac{1}{2} F_{a b}^{i j}\left(x_{0}\right) \psi_{0}^{a} \psi_{0}^{b}
$$

Collecting from (3.54), (3.56) and (3.57) we obtain, for the exponent

$$
\frac{i}{n}\left(S-i \bar{\xi}_{1} \Delta \xi-i \bar{\eta}_{1} \Delta \eta\right)=\frac{i \alpha p}{2}+\frac{i}{2} f^{i i} t+\frac{1}{n} \bar{\eta}_{t}(\exp (i \alpha+i \xi t)-1) \eta_{0}+0(\beta)
$$

Note that, for practical reasons, we are using the variables $\psi_{0}$ instead of $\bar{\xi}_{t} \xi_{0}$. We will take care of this by integrating over $\psi_{0}$ rather than $\bar{\xi}_{t} \xi_{0}$, with the appropriate Jacobian. 
The superdeterminant $D^{\prime}$ is obtained from eq. (2.26) by using $(\xi, \eta)$ instead of $\theta$

$$
D^{\prime}=(-)^{3 / 2 n+p} \text { Sdet }\left\|\begin{array}{ccc}
\frac{\partial^{2} S^{\prime}}{\partial x_{t} \partial x_{0}} & \frac{\partial^{2} S^{\prime}}{\partial x_{t} \partial \xi_{0}} & \frac{\partial^{2} S^{\prime}}{\partial x_{t} \partial \eta_{0}} \\
i \frac{\partial^{2} S^{\prime}}{\partial \bar{\xi}_{t} \partial x_{0}} & i \frac{\partial^{2} S^{\prime}}{\partial \bar{\xi}_{t} \partial \xi_{0}} & i \frac{\partial^{2} S^{\prime}}{\partial \bar{\xi}_{t} \partial \eta_{0}} \\
i \frac{\partial^{2} S^{\prime}}{\partial \bar{\eta}_{t} \partial x_{0}} & i \frac{\partial^{2} S^{\prime}}{\partial \bar{\eta}_{t} \partial \xi_{0}} & i \frac{\partial^{2} S^{\prime}}{\partial \bar{\eta}_{t} \partial \eta_{0}}
\end{array}\right\|
$$

By equation (2.21), a general variation of $S^{\prime}$ at constant $t$ is

$$
\delta S^{\prime}=\delta x_{1} p_{t}+i \xi_{t} \delta \bar{\xi}_{t}+i \eta_{t} \delta \bar{\eta}_{t}-\delta x_{0} p_{0}+i \delta \xi_{0} \bar{\xi}_{0}+i \delta \eta_{0} \bar{\eta}_{0}
$$

This last equation may be used to express the superdeterminant in the following more convenient way

$$
D^{\prime}=(-)^{3 / 2 n+\nu} \text { sdet }\left\|\begin{array}{ccc}
-\frac{\partial p_{0}}{\partial x_{t}} & \frac{\partial p_{t}}{\partial \xi_{0}} & \frac{\partial p_{t}}{\partial \eta_{0}} \\
-i \frac{\partial p_{0}}{\partial \bar{\xi}_{t}} & -\frac{\partial \xi_{t}}{\partial \xi_{0}} & -\frac{\partial \xi_{t}}{\partial \eta_{0}} \\
-i \frac{\partial p_{0}}{\partial \bar{\eta}_{t}} & -\frac{\partial \eta_{t}}{\partial \xi_{0}} & -\frac{\partial \eta_{t}}{\partial \eta_{0}}
\end{array}\right\|
$$

where all quantities differentiated are considered functions of

$$
\left(x_{1}, \bar{\xi}_{t}, \bar{\eta}_{1}, x_{0}, \bar{\xi}_{0}, \bar{\eta}_{0} ; t\right)
$$

and the derivatives are evaluated for $x_{1}=x_{0}$. From (3.50) and (3.21) with (3.52) the following orders in $\beta$ are immediately obtained for the above su- 
permatrix

$$
\left(\begin{array}{lll}
0\left(\beta^{-1}\right) & 0\left(\beta^{1 / 2}\right) & 0(1) \\
0\left(\beta^{1 / 2}\right) & -1+0(\beta) & 0\left(\beta^{1 / 2}\right) \\
0(1) & 0\left(\beta^{1 / 2}\right) & 0(1)
\end{array}\right)
$$

With this, the general expression for the superdeterminant, eq. (E.4), yields

$$
D^{\prime}=\operatorname{det}\left(\frac{\partial p_{0}}{\partial x_{t}}\right) \cdot \operatorname{det}\left(\frac{\partial \eta_{t}}{\partial \eta_{0}}\right)^{-1}+0\left(\beta^{-n+1}\right)
$$

To compute the derivative of $p_{0}$ with respect to $x_{\ell}$ only the first term in eq. (3.53a) needs to be kept, since the others give contributions which, to leading order in $\beta$, are independent of $p_{0}$.

$$
\bar{x}^{\mu} \simeq \frac{i}{2} R^{\mu}{ }_{\text {nab }}\left(x_{0}\right) \psi_{0}^{a} \psi_{0}^{b} \dot{x}^{\nu}+0(1)
$$

Defining

$$
R_{\nu}^{\mu} \equiv \frac{1}{2} R_{\operatorname{vab}}^{\mu}\left(x_{0}\right) \psi_{0}^{a} \psi_{0}^{b}
$$

we obtain

$$
x_{t}^{\mu} \simeq\left(\frac{\exp (i R t)-1}{i R}\right)^{\nu} \nu g^{\nu a} p_{0 a}+o\left(\beta^{2}\right)
$$

and [16]

$$
\operatorname{det}\left(\frac{\partial p_{0}}{\partial x_{t}}\right)=\left(\operatorname{det}\left(\frac{\partial x_{t}}{\partial p_{0}}\right)\right)^{-1}=\frac{g\left(x_{0}\right)}{t^{n}} \operatorname{det} \frac{i t R / 2}{\sinh (i t R / 2)}+0\left(\beta^{-n+1}\right)
$$

From eq. (3.57) we have

$$
\operatorname{det}\left(\frac{\partial \eta_{t}}{\partial \eta_{0}}\right)^{-1}=e^{-i-0} \operatorname{det}(\exp (-i \bar{f} t))+0(\beta)
$$

and $\sqrt{D^{\prime}}$ is given by

$$
\sqrt{D^{\prime}}=\frac{\sqrt{g\left(z_{0}\right)}}{t^{n / 2}} \sqrt{\operatorname{det} \frac{i t R / 2}{\sinh (i t R / 2)}} e^{-i p a / 2} \cdot \exp \left(\frac{-i F^{i i} t}{2}\right)+0\left(\beta^{-n / 2+1}\right)
$$


where we have used the identity

$$
\operatorname{det}(\exp (-i \mp t))=\exp \left(-i \mathcal{F}^{i i} t\right)
$$

The index density is obtained by performing the fermionic integrals in (3.31). Since both the exponent and the integrand are written in terms of $\psi_{0}$ rather than $\bar{\xi}_{t}$ and $\xi_{0}$, we have to change the integration variables and introduce a Jacobian

$$
\int_{\xi_{0} \eta_{0}} \int_{\bar{F}_{1} \bar{\xi}_{t}}=\int_{\varepsilon_{0} \bar{\xi}_{0}} \int_{\eta_{0} \bar{\eta}_{0}} \operatorname{det}\left(\frac{\partial \bar{\xi}_{0}}{\partial \bar{\xi}_{t}}\right)=(-i)^{n / 2} \int_{\phi_{0}} \int_{\eta_{0} \bar{\eta}_{t}} \operatorname{det}\left(\frac{\partial \bar{\xi}_{0}}{\partial \bar{\xi}_{t}}\right)
$$

where

$$
\int_{\phi_{0}} \equiv \int_{\phi_{0}^{n} \nu_{0}^{n-1} \ldots \nu_{0}^{2}}
$$

The determinant in (3.73) can be neglected because by eq. (3.43)

$$
\operatorname{det}\left(\frac{\partial \bar{\xi}_{0}}{\partial \bar{\xi}_{1}}\right)=1+0(\beta)
$$

Only the exponent in (3.31) depends on $\bar{\eta}_{f}$ and $\bar{\eta}_{0}$, and the integral over these variables gives

$$
(-n)^{p} \int_{\eta_{0} \eta_{1}} \exp \left(\frac{i}{h} \pi_{1}(\exp (i \alpha+i f t)-1) \eta_{0}\right)=\operatorname{det}(1-\exp (i \alpha+i \xi t))
$$

and eq. (3.31) for $I(\alpha)$ becomes

$I(\alpha)=\lim _{t \rightarrow 0}\left\{\frac{1}{(2 \pi)^{n / 2}} \int_{\Sigma_{0}} d x_{0} \frac{\sqrt{g\left(x_{0}\right)}}{t^{n / 2}} \int_{\psi_{0}} \sqrt{\operatorname{det} \frac{i R t / 2}{\sinh (i R t / 2)}} \operatorname{det}(1-\exp (i \alpha+i \xi t))\right\}$

Only the term proportional to $t^{n / 2}$ survives the fermionic integration, thus cancelling the $t^{n / 2}$ in the denominator. Using

$$
\int_{3} d x \sqrt{\operatorname{det} g(x)} \int_{\psi} C_{a b c \ldots} \psi^{a} \psi^{b} \psi^{c} \ldots=\int_{x}{ }^{b} b^{\mu} d x^{\nu} d x^{\lambda} \ldots C_{\mu \nu \lambda \ldots}
$$


we can write $I(\alpha)$ in the following form

$$
I(\alpha)=\int_{x} \sqrt{\operatorname{det} \frac{i \Re / 4 \pi}{\sinh (i \Re / 4 \pi)}} \operatorname{det}\left(1-e^{i \alpha} e^{i O t / 2 \pi}\right)
$$

where $\Re$ and $\Im$ ase now the followiri (matrix valued) two forms

$$
\begin{aligned}
& \Re_{\nu}^{\mu} \equiv \frac{1}{2} R_{\nu \alpha \beta}^{\mu}(x) d x^{\alpha} d x^{\beta} \\
& \Im^{i j} \equiv \frac{1}{2} F_{\alpha \theta}^{i j}(x) d x^{\alpha} d x^{\rho}
\end{aligned}
$$

Expanding

$$
\operatorname{det}\left(1-e^{i a} e^{i Q / 2 \pi}\right)=1-e^{i a} \operatorname{tr} e^{i S / 2 \pi}+\ldots
$$

we finally get the standard formula for the index [17]

$$
N_{+}-N_{-}=I_{1}=\int_{2} \sqrt{\operatorname{det} \frac{i N / 4 \pi}{\sinh (i \Re / 4 \pi)}} \cdot \operatorname{tr} e^{i 0 / 2 \pi}
$$




\section{Appendix A}

We present here explicit expressions for some of the objects defined in sect. 1.1. These expressions are taken from $[4,5]$ and adapted to the conventions used in chapter 1.

For $\omega_{2 n-3}^{1}(A, v)$ we have

$$
w_{2 n-2}^{1}(A, v)=n(n-1) \int_{0}^{1} \delta t(1-t) \operatorname{Str}\left(v d\left(A_{t}^{n-2}\right)\right)
$$

Here $F_{t}=d A_{t}+A_{t}^{2}=t d A+t^{2} A^{2}$, and $S t r$ is a "symmetrized trace". The symmetrized trace of a product of Lie algebra valued forms $\Lambda_{i}$ is defined by

$$
\operatorname{Str}\left(\Lambda_{1}, \Lambda_{2}, \ldots, \Lambda_{n}\right)=\frac{1}{n !} \sum_{\left(i_{1}, \ldots, i_{n}\right)} \operatorname{sgn}\left(i_{1}, \ldots, i_{n}\right) \operatorname{tr}\left(\Lambda_{i_{1}}, \ldots, \Lambda_{i_{n}}\right)
$$

where the sum is over all permutations $\left(i_{1}, \ldots, i_{n}\right)$ of $(1,2, \ldots, n)$ and $\operatorname{sgn}\left(i_{1}, \ldots, i_{n}\right)$ is the sign arising from the commutative properties of $\Lambda_{i}$ as forms.

$\alpha_{2 n-2}$ is given by

$$
\alpha_{2 n-2}(A, g)=-n(n-1) \iint \operatorname{Str}\left(V A F_{\lambda \mu}^{m-2}\right)
$$

where $V \equiv d g g^{-1}, F_{\lambda \mu}=d A_{\lambda \mu}+A_{\lambda \mu}^{2}$ and $A_{\lambda \mu}=\lambda A-\mu V$. The integral is over a triangle in the parameter space $(\lambda, \mu)$ :

$$
\iint=\int_{0}^{1} \delta \lambda \int_{0}^{1-\lambda} \delta \mu
$$

In the evaluation of (A.3) all the integrals are of the type

$$
\iint \lambda^{h} \mu^{k}=\frac{h ! k !}{(h+k+2) !}
$$


For $n=3($ A.1) gives

$$
\omega_{4}^{1}(A, v)=\operatorname{tr}\left\{v d\left(A d A+\frac{1}{2} A^{3}\right)\right\}
$$

and we can express the variation of the functional $W(R, L, U)$ as follows:

$$
\begin{aligned}
\delta_{v} W(R, L, U) & =K_{3} \int_{D_{s}} \delta \omega_{3}^{0}(R, L)=K_{3} \int_{S^{*}} \omega_{4}^{1}\left(R, v_{R}\right)-\omega_{4}^{1}\left(L, v_{L}\right) \\
& =\frac{-i}{24 \pi^{2}} \int_{S^{*}} \operatorname{tr}\left\{v_{R} d\left(R d R+\frac{1}{2} R^{3}\right)-(R \leftrightarrow L)\right.
\end{aligned}
$$

in agreement with Witten's results $[10]$ and computations at the quark level [2]. 


\section{Appendix B}

Here we complete the proof of eqs. (1.57) and (1.59) which give $\tilde{\omega}_{2 n-1}^{0}$ and $S_{2 n-1}$. To show the invariance of (1.57) under a vector gauge transformation we have to notice that, for $g_{R}=g_{\mathrm{L}}=g=e^{A}, V_{+}$and $V_{-}$transform in the same way:

$$
V_{ \pm} \rightarrow g^{-1} V_{ \pm} g+g^{-1} d g
$$

which can be written to first order in $\beta$

$$
\delta_{\beta} V_{ \pm}=\left[V_{ \pm}, \beta\right]+d \beta
$$

Also, under global (space-time independent) vector gauge transformation the integrand is obviously invariant, and we only have to consider the part of the variation proportional to $d \beta$, which we indicate by writing $\sim$ instead of $=$ :

$$
\delta_{\beta} V_{+} \sim \delta_{\beta} V_{-} \sim d \beta
$$

Since $\delta_{\beta} F_{t}=\left[F_{t}, \beta\right]$, we have

$$
\delta_{\rho} F_{+} \sim \delta_{\rho} F_{-} \sim 0
$$

On the line $\lambda+\mu=1, \delta A_{\lambda \mu}$ is

$$
\delta A_{\lambda \mu}=\delta \lambda\left(V_{+}-V_{-}\right)
$$

and

$$
\delta_{\beta}\left(\delta A_{\lambda \mu}\right) \sim \delta \lambda(d \beta-\delta \beta)=0
$$

$F_{\lambda \mu}$ can be written

$$
F_{\lambda \mu}=\lambda\left\{F_{+}+(\lambda-1) V_{+}^{2}\right\}+\mu\left\{F_{-}+(\mu-1) V_{-}^{2}\right\}+\lambda \mu\left(V_{+} V_{-}+V_{-} V_{+}\right)
$$


From (B.3), (B.4) and (B.7) it follows immediately that, on the line $\lambda+\mu=1$, $\delta_{\beta} F_{\lambda \mu} \sim 0$.

This completes the proof of eq. (1.57). Let us consider now the expression (1.58) for $d S_{2 n-2}$

$$
\begin{aligned}
& d S_{2 n-2}=\frac{n}{D} \int \operatorname{Str}\left(\gamma_{5} \delta A_{\lambda_{\mu}} F_{\lambda_{\mu}}^{n-1}\right) \\
& =\frac{n}{D} \int \delta \lambda \operatorname{Str}\left(\gamma_{s} V_{+} F_{\lambda \mu}^{n-1}\right)+\delta \mu \operatorname{Str}\left(\gamma_{5} \delta V_{-} F_{\lambda \mu}^{n-1}\right)
\end{aligned}
$$

This expression is transformed by using Stokes theorem into an integral over the triangle of fig. 2:

$$
d S_{2 n-2}=\frac{n}{D} \iint \operatorname{Str} \gamma_{3}\left(V_{+} \frac{\partial}{\partial \mu}-V_{-} \frac{\partial}{\partial \lambda}\right) F_{\lambda \mu}^{n-1}
$$

Note that in (B.8) we are using the symmetrized trace, which in this case coincides with the ordinary one, but is more convenient in what follows. Using

$$
\frac{\partial F_{\lambda \mu}}{\partial \lambda}=d V_{+}+\left\{A_{\lambda \mu}, V_{+}\right\}, \quad \frac{\partial F_{\lambda \mu}}{\partial \mu}=d V_{-}+\left\{A_{\lambda \mu}, V_{-}\right\}
$$

we can write (B.9) as

$$
\begin{aligned}
d S_{2 n-2} & =\frac{n(n-1)}{D} \iint \operatorname{Str} \gamma_{5}\left(\left(V_{+} d V_{-}-V_{-} d V_{+}\right) F_{\lambda_{\mu}}^{n-2}\right. \\
& \left.+V_{+}\left\{A_{\lambda \mu}, V_{-}\right\} F_{\lambda_{\mu}-2}^{m-V}\left\{A_{\lambda_{\mu}}, V_{+}\right\} F_{\lambda_{\mu}}^{n-2}\right)
\end{aligned}
$$

Since the symmetrized trace is invariant under permutations (up to a sign due to the commutation properties of the forms involved) the last two terms can be combined

$$
\begin{aligned}
& \operatorname{Str} \gamma_{5}\left(\left\{A_{\lambda \mu}, V_{-}\right\} V_{+} F_{\lambda_{\mu}}^{n-2}-V_{-}\left\{A_{\lambda \mu}, V_{+}\right\} F_{\lambda \mu}^{n-2}\right) \\
& =-\operatorname{Str}\left(\gamma_{5} V_{-} V_{+}\left[A_{\lambda_{\mu}}, F_{\lambda_{\mu}}^{n-2}\right]\right)=\operatorname{Str}\left(\gamma_{5} V_{-} V_{+} d F_{\lambda \mu}^{n-1}\right)
\end{aligned}
$$


In the last step the Bianchi identity for $F_{\lambda \mu}$ has been used. This allows (B.11) to be written as a total differential

$$
d S_{2 n-2}=\frac{n(n-1)}{D} d \iint \operatorname{Str}\left(\gamma_{5} V_{-} V_{+} F_{\lambda \mu}^{n-2}\right)
$$

and

$$
S_{2 n-2}=\frac{n(n-1)}{D} \iint \operatorname{Str}\left(\gamma_{8} V_{-} V_{+} F_{\lambda \mu}^{n-2}\right)
$$

The transformations leading from (B.8) to (B.14) are identical to the ones used by Zumino to prove eq. (A.3) for $\alpha_{2 n-3}$.

Notice the similarity of eqs. (A.3) and (B.24), which up to a factor differ only by the introduction of a $\gamma_{5}$ and the substitution $V_{-} \leftrightarrow-V$. Nevertheless, in the process of evaluating $\alpha_{2 n-2}$ from (A.3) the equality $d V=V^{2}$ is used. this equality does not hold for $V_{-}$, and as a consequence the final expression for $S_{2 n-2}$ cannot be obtained simply from that for $\alpha_{2 n-2}$, but has to be evaluated directly from (B.14). 


\section{Appendix C}

In this appendix we prove the continuity equation for the Van Vleck superdeterminant, eq. (2.15).

The supermatrix of second derivatives of the classical action

$$
S_{i j} \equiv \frac{\partial^{2} S}{\partial Q_{t i} \partial Q_{0 j}} \quad ; \quad i, j: 1 \ldots N
$$

can be decomposed into four submatrices

$$
\left(S_{i j}\right)=\left(\begin{array}{cc}
A & \Gamma \\
\Delta & B
\end{array}\right)
$$

such that the indices $i, j$ are bosonic in $A$, fermionic in $B$ and of opposite nature in $\Gamma$ and $\Delta$. The supertrace is defined by

$$
\operatorname{Str}\left(S_{i j}\right) \equiv \operatorname{tr} A-\operatorname{tr} B \equiv \sigma(i) S_{i j}
$$

where $\sigma(i)$ has been defined in eq. (2.8). The superdeterminant is [26]

$$
D=\operatorname{Sdet}\left(S_{i j}\right)=\frac{\operatorname{det}\left(A-\Gamma B^{-1} \Delta\right)}{\operatorname{det} B}
$$

and satisfies the usual properties of an ordinary determinant. In particular

$$
\delta \operatorname{Sdet} S_{i j}=\operatorname{Sdet}\left(S_{i j}\right) \cdot \operatorname{Str}\left(S_{i j}^{-1} \delta S_{j k}\right)
$$

To prove the continuity equation, we first differentiate the HamiltonJacobi eq. (2.12) with respect to $Q_{O_{i}}$

$$
\frac{\partial^{2} S}{\partial t \partial Q_{0 i}}+\frac{\partial^{2} S}{\partial Q_{0 i} \partial Q_{1 \ell}} \frac{\partial H}{\partial P_{i \ell}}=0
$$

where we have used

$$
P_{l \ell}=\frac{\partial S}{\partial Q_{l \ell}}
$$


Differentiating again with respect to $Q_{t k}$ yields

$\frac{\partial}{\partial t}\left(\frac{\partial^{2} S}{\partial Q_{t k} \partial Q_{0 i}}\right)+\frac{\partial^{3} S}{\partial Q_{t k} \partial Q_{0 i} \partial Q_{i \ell}} \frac{\partial H}{\partial P_{t \ell}}+\sigma(i, k) \sigma(\ell, k) \frac{\partial^{2} S}{\partial Q_{0 i} \partial Q_{t \ell}} \frac{\partial}{\partial Q_{t k}}\left(\frac{\partial H}{\partial P_{t \ell}}\right)=0$

The symbols $\sigma(i, k)$ and $\sigma(l, k)$ take care of the anticommutation properties of the fermionic variables and have been defined in eq. (2.43). This last equation may also be written

$$
\frac{\partial S_{k i}}{\partial t}+\sigma(\ell, k) \sigma(\ell, i) \frac{\partial S_{k i}}{\partial Q_{l \ell}} \frac{\partial H}{\partial P_{l \ell}}+\sigma(i, k) \sigma(\ell, k) \sigma(i, \ell) S_{\Delta i} \frac{\partial}{\partial Q_{t L}}\left(\frac{\partial H}{\partial P_{t \ell}}\right)=0
$$

If we multiply by $\sigma(i) S_{i k}^{-1}$ and sum over repeated indices, by eq. (C.5) the first term gives

$$
\sigma(i) S_{i k}^{-1} \frac{\partial S_{k i}}{\partial t}=\frac{1}{D} \frac{\partial D}{\partial t}
$$

and the second one

$$
\sigma(i) \sigma(\ell, k) \sigma(l, i) S_{i k}^{-1} \frac{\partial S_{k i}}{\partial Q_{i \ell}} \frac{\partial H}{\partial P_{t \ell}}=\frac{1}{D} \frac{\partial D}{\partial Q_{u \ell}} \frac{\partial H}{\partial P_{t \ell}}
$$

where we have used eq. (C.5) with

$$
\sigma(\ell, k) \sigma(\ell, i) \delta Q_{1 \ell} S_{i k}^{-1} \frac{\partial S_{k i}}{\partial Q_{1 \ell}}=S_{i k}^{-1} \delta S_{k i}
$$

The last term becomes

$$
\delta_{k e} \frac{\partial}{\partial Q_{t h}} \frac{\partial H}{\partial P_{t e}}=\frac{\partial}{\partial Q_{u}} \frac{\partial H}{\partial P_{t l}}
$$

because

$$
S_{b} S_{i k}^{-1}=\delta_{k e}
$$

implies

$$
\sigma(i) \sigma(\ell, i) \sigma(\ell, k) \sigma(i, k) S_{i k}^{-1} S_{\ell i}=\delta_{k \ell}
$$


After multiplying by $D$, adding (C.10), (C.11) and (C.13), we get

$$
\frac{\partial D}{\partial t}+\frac{\partial}{\partial Q_{t \ell}}\left(D \frac{\partial H}{\partial P_{t \ell}}\right)=0
$$

The continuity equation (2.25) for $\bar{D}$ is obtained in the same way, but instead of eq. (C.6) we have

$$
\begin{gathered}
\frac{\partial^{2} \dot{S}}{\partial t \partial Q_{0 k}}+\frac{\partial^{2} \dot{S}}{\partial Q_{0 k} \partial Q_{t i}} \frac{\partial H}{\partial P_{t i}}-\sigma(j) \frac{\partial^{2} \dot{S}}{\partial Q_{0 k} \partial P_{t j}} \frac{\partial H}{\partial Q_{t j}}=0 \\
k: 1, \ldots, N \quad i: 1, \ldots, R \quad j: R+1, \ldots, N
\end{gathered}
$$

which explains the $-\sigma(j)$ sign in eq. (2.25). 


\section{Appendix D}

Here we clarify some of the definitions and conventions used in the fermionic integrals in this paper. We always use the "nested integration" convention

$$
\int_{\phi_{1} \ldots \phi_{n}} \phi_{n} \ldots \phi_{1}=1, \forall n
$$

When integrating over complex variables $\theta_{i}$ and $\bar{\theta}_{i}$, the following abbreviations are used

$$
\int_{0} \equiv \int_{o_{1} \ldots o_{m}} ; \int_{\overline{0}} \equiv \int_{\overline{0}_{m} \ldots \overline{0}_{1}}
$$

which imply

$$
\begin{gathered}
\int_{0} \int_{\bar{\theta}}=\int_{0 \bar{i}}=\int_{0_{i} \bar{D}_{1} \ldots \theta_{m} \bar{J}_{m}} \\
\int_{0 \overline{0}} \exp \left(\bar{\theta}_{i} M_{i j} \theta_{j}\right)=\operatorname{det} M
\end{gathered}
$$

For real variables $\psi^{\mu}$ we define

$$
\int_{\downarrow} \equiv \int_{\downarrow n \ldots \downarrow^{2}}
$$

so that

$$
\int d x \int_{\downarrow} C_{\mu \nu \ldots . . .} \psi^{\mu} \psi^{\nu} \psi^{\rho} \ldots \equiv \int C_{\mu \omega_{\ldots} \ldots} d x^{\mu} d x^{\nu} d x^{\rho} \ldots
$$

for any completely antisymmetric tensor $C$.

The Fourier transform is taken to be (for $i: 1 \ldots m$ )

$$
f(\theta)=\int_{\bar{\theta}} e^{-\overline{\bar{\theta}}_{i} \boldsymbol{l}_{i}} \dot{f}(\bar{\theta}) ; \dot{f}(\bar{\theta})=(-)^{m} \int_{0} e^{\bar{j}_{i} \theta_{i}} f(\theta)
$$

The $\delta$-function is defined by

$$
f(\xi)=\int_{0} \delta(\xi-\theta) f(\theta), \delta(\xi-\theta)=\left(\theta_{m}-\xi_{m}\right) \cdots\left(\theta_{1}-\xi_{1}\right)
$$


and its Fourier transform is

$$
\hat{\delta}(\bar{\xi}, \theta)=e^{\bar{\xi} \theta}
$$

If we define the kernel $K$ of a fermionic system by

$$
\Psi\left(\theta_{i} ; t\right)=\int_{\theta_{0}} K\left(\theta_{t}, \theta_{0} ; t\right) \Psi\left(\theta_{0} ; 0\right)
$$

and another kernel $\dot{K}$ by

$$
\dot{\Psi}\left(\bar{\theta}_{t} ; t\right)=\int_{0_{0}} \dot{K}\left(\bar{\theta}_{t}, \theta_{0} ; t\right) \Psi\left(\theta_{0} ; 0\right)
$$

then eqs. (D.6) imply

$$
\begin{aligned}
\int_{0_{0}} \tilde{K}\left(\bar{\theta}_{t}, \theta_{0} ; t\right) \Psi\left(\theta_{0}, 0\right) & =(-)^{m} \int_{0_{1}} e^{{\overline{j_{1}}}_{1} \theta_{1}} \Psi\left(\theta_{t} ; t\right) \\
& =(-)^{m} \int_{0_{1}} \int_{0_{0}} e^{\bar{j}_{1} \theta_{1}} K\left(\theta_{t}, \theta_{0} ; t\right) \Psi\left(\theta_{0} ; 0\right) \\
& =\int_{0_{0}} \int_{0_{1}} e^{\bar{\theta}_{1} \theta_{1}} K\left(\theta_{t}, \theta_{0} ; t\right) \Psi\left(\theta_{0} ; 0\right)
\end{aligned}
$$

and we have

$$
\bar{K}\left(\bar{\theta}_{1}, \theta_{0} ; t\right)=\int_{0_{1}} e^{\bar{\theta}_{1} \theta_{1}} K\left(\theta_{1}, \theta_{0}, t\right) \equiv(-)^{m} \dot{K}\left(\bar{\theta}_{1}, \theta_{0} ; t\right)
$$

We see that $\dot{K}$ is equal to the Fourier transform of $K$ only up to a sign $(-)^{m}$. Thus, the correct limit of $\ddot{K}$ as $t \rightarrow 0$ is

$$
\lim _{i \rightarrow 0} \bar{K}\left(\bar{\theta}_{i}, \theta_{0} ; t\right)=(-)^{m} e^{\bar{i}_{1} \nu_{0}}
$$

This explains the value of the constant $C^{\prime}$ in eq. (2.57).

The set of $2^{m}$ wave functions

$$
1, \theta_{1}, \theta_{2}, \ldots, \theta_{1} \theta_{2}, \theta_{1} \theta_{3}, \ldots, \theta_{1} \theta_{2} \theta_{3} \ldots \theta_{m}
$$


constitutes an orthonormal basis with sespect to the inner product

$$
\left\langle\Psi_{1} \mid \Psi_{2}\right\rangle=\int_{0 \bar{\theta}} e^{\bar{\theta} \theta} \Psi_{1}^{*}(\bar{\theta}) \Psi_{2}(\theta)
$$

with the vacuum represented by

$$
|0\rangle=\theta_{1} \ldots \theta_{m}, \hat{\theta}_{i}|0\rangle=\theta_{i}|0\rangle=0 \quad \forall i: 1 \ldots m
$$

To obtain formulae for the trace and supertrace of the evolution operator $\exp (-i t \hat{y})$ we first consider two identities. Denoting by $\epsilon_{i}(\theta)$ the elements in the orthonormal basis (D.12) we have, for any wave function $\Psi(\theta)$

$$
\begin{aligned}
\Psi(\theta)=e_{i}(\theta) a_{i} & =e_{i}(\theta)\left\langle e_{i} \mid \Psi\right\rangle=e_{i}(\theta) \int_{\xi \bar{G}} e^{\bar{\xi} \xi} e_{i}^{*}(\bar{\xi}) \Psi(\xi) \\
& =\sigma(i) \sigma(i, \Psi) \int_{\bar{G}_{\bar{G}}} e^{\bar{\xi} \xi} e_{i}^{*}(\bar{\xi}) \Psi(\xi) e_{i}(\theta)
\end{aligned}
$$

This is the first identity. The second is obtained by observing that $e_{i}(-\theta)=$ $\sigma(i) e_{i}(\theta)$

$$
\Psi(-\theta)=\sigma(i, \Psi) \int_{\xi \bar{\xi}} e^{\bar{\varepsilon} \xi} e_{i}^{i}(\bar{\xi}) \Psi(\xi) e_{i}(\theta)
$$

Then the trace of the evolution operator is

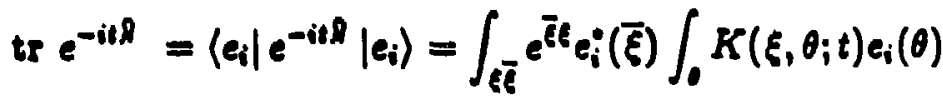

$$
\begin{aligned}
& =\int_{0} \sigma(i, K) \int_{\xi \bar{\varepsilon}} e^{\bar{\xi} \xi} e_{i}^{-}(\bar{\xi}) K(\xi, \theta ; t) e_{i}(\theta)=\int_{0} K(-\theta, \theta ; t)(D .17)
\end{aligned}
$$

where the identity (D.16) has been used in the last step .

The supertrace is defined by

$$
\begin{aligned}
\operatorname{Str} e^{-i t \hbar} & \equiv \operatorname{tr}(-)^{\theta} e^{-i t \theta}=(-)^{m} \sigma(i)\left\langle e_{i}\left|e^{-i t \pi}\right| e_{i}\right\rangle \\
\hat{F} & =\dot{\theta}_{i} \theta_{i}
\end{aligned}
$$


The sign $(-)^{m}$ guarantees that the vacuum (D.14) is always assigned a plus sign. Then, by using the identity (D.15) we obtain

$$
\operatorname{Str} e^{-i t \theta} \equiv \operatorname{tr}(-)^{\hat{f}} e^{-i t \theta}=(-)^{m} \int_{0} K(\theta, \theta ; t)
$$

By adding an integration over bosonic coordinates, wherever they are present, eqs. (D.13), (D.18) and (D.19) become (2.30), (2.64) and (2.67), respectively. 


\section{Appendix E}

In this appendix we study the behavior as $t \rightarrow 0$ of the modified classical action $S^{\prime}\left(x_{t}, \bar{\theta}_{t}, x_{0}, \theta_{0} ; t\right)$ for the following Lagrangian

$$
\mathcal{L}=\frac{1}{2} g_{\mu \nu}(x) \dot{x}^{\mu} \dot{x}^{\nu}+i \bar{\theta}_{i} \dot{\theta}_{i}+\dot{x}^{\mu} B_{\mu}(x, \theta, \bar{\theta})-W(x, \theta, \bar{\theta})
$$

where $B_{\mu}$ and $W$ are analytic superfunctions and $g \equiv \operatorname{det}\left|g_{\mu \nu}\right| \neq 0$. The equations of motion are

$$
\begin{gathered}
\dot{x}^{\mu}+\Gamma_{a, \rho^{\alpha}}^{\mu} \dot{x}^{\rho}=g^{\mu \nu}\left(B_{\alpha, \nu}-B_{\mu, \alpha}\right) \dot{x}^{\alpha}-g^{\mu \nu}\left(B_{\nu, i} \dot{\theta}_{i}+B_{\mu, i} \dot{\bar{\theta}}_{i}\right)-W^{, \mu} \\
\dot{\theta}_{i}+\dot{x}^{\mu} B_{\mu, i}-W_{, i}=0 \\
i \dot{\bar{\theta}}_{i}+\dot{x}^{\mu} B_{\mu, i}-W_{, i}=0
\end{gathered}
$$

where

$$
B_{\mu, i} \equiv \frac{\partial B_{\mu}}{\partial \bar{\theta}_{i}} ; \quad B_{\mu, i} \equiv \frac{\partial B_{\mu}}{\partial \theta_{i}}
$$

We make the following rescaling

$$
t^{\prime}=t r, 0 \leq t^{\prime} \leq t, 0 \leq r \leq 1
$$

and in what follows dots denote derivatives with respect to $r$. We consider the solutions to (E.2) expanded in powers of $t$

$$
\begin{aligned}
& x\left(t^{\prime}\right)=y_{0}(\tau)+t y_{1}(\tau)+t^{2} y_{2}(\tau)+\ldots \\
& \theta\left(t^{\prime}\right)=\varphi_{0}(\tau)+t \varphi_{1}(\tau)+t^{2} \varphi_{2}(\tau)+\ldots \\
& \bar{\theta}\left(t^{\prime}\right)=\bar{\varphi}_{0}(\tau)+t \bar{\varphi}_{1}(\tau)+t^{2} \bar{\varphi}_{2}(\tau)+\ldots
\end{aligned}
$$


The integral of $\mathcal{L}$ along the classical path can also be expanded in powers of $t$, and we get

$$
\begin{aligned}
S & =\int_{0}^{1} \mathcal{L} d t^{\prime}=\frac{1}{2 t} \int_{0}^{1} g_{\mu \nu}\left(y_{0}\right) \dot{y}_{0}^{\mu} \dot{y}_{0}^{\mu} d \tau+i \int_{0}^{1} \bar{\varphi}_{0 i} \dot{\varphi}_{0 i} d \tau \\
& +\int_{0}^{1} \dot{y}_{0}^{\mu} B_{\mu}\left(y_{0}, \varphi_{0}, \bar{\varphi}_{0}\right) d(\tau)+0(t)
\end{aligned}
$$

Thus, only $y_{0}, \varphi_{0}$ and $\bar{\varphi}_{0}$ have to be determined in order to obtain the behavior of the action as $t \rightarrow 0$. Rescaling the equations of motion and substituting the expansions (E.5) into them we get

$$
\begin{aligned}
& \bar{y}_{0}^{\mu}+\Gamma_{a,}^{\mu}\left(y_{0}\right) y_{0}^{\alpha} y_{0}^{\rho}=0 \\
& i \dot{\varphi}_{0 a}+\dot{y}^{\mu} B_{\mu_{1} \mathrm{~d}}\left(y_{0}\right)=0 \\
& i \dot{\varphi}_{0 a}+\dot{y}^{\mu} B_{\mu_{1} a}\left(y_{0}\right)=0
\end{aligned}
$$

This shows that, as for purely bosonic systems [29], the coefficient of $1 / t$ in the classical action is given by $(1 / 2)$ the geodesic distance between $x_{0}$ and I. Integration of (E.7a) yields

$$
y^{\mu}(\tau)=y^{\mu}(0)+\dot{y}^{\mu}(0) \tau-\frac{1}{2} \Gamma_{\alpha \rho}^{\mu}(0) \dot{y}^{\alpha}(0) \dot{y}^{\rho}(0) \tau^{2}+\left(\tau^{J}\right)
$$

The boundary conditions are

$$
y^{\mu}(0)=x_{0}^{\mu} \quad y^{\mu}(1)=x_{t}^{\mu}
$$

which imply

$$
\begin{aligned}
\dot{y}_{0}^{\mu}(0) & =X^{\mu}+\frac{1}{2} \Gamma_{a \rho}^{\mu}\left(x_{0}\right) X^{\alpha} X^{\theta}+O\left(X^{3}\right) \\
X^{\mu} & \equiv x_{f}^{\mu}-x_{0}^{\mu}
\end{aligned}
$$


and we have

$$
\frac{1}{2 t} \int_{0}^{t} g_{\mu \nu}\left(y_{0}\right) \dot{y}_{0}^{\mu} \dot{y}_{0}^{\nu} d \tau=\frac{1}{2 t}\left(g_{\mu \nu}\left(x_{0}\right) X^{\mu} X^{\nu}+O\left(X^{3}\right)\right)
$$

If we set $X^{\mu} \equiv x_{t}^{\mu}-x_{0}^{\mu}=0$, then the solution to (E.7) is

$$
\begin{array}{r}
y_{0}^{\mu}(\tau)=0, \varphi_{0}(\tau)=\varphi_{0}(0)=\theta_{0} \\
\bar{\varphi}_{0}(\tau)=\bar{\varphi}_{0}(0)=\bar{\theta}_{0}
\end{array}
$$

This shows that the second and third terms in (E.6) vanish for $X^{\mu}=0$, and we can write

$$
\int_{0}^{t} \mathcal{L} d t^{\prime}=\frac{1}{2 t}\left(g_{\mu \nu}\left(x_{0}\right) X^{\mu} X^{\nu}+0\left(X^{3}\right)\right)+O(X)+0(t)
$$

The modified classical action $S^{\prime}$ is

$$
S^{\prime}=S(\cdot)-i \bar{\theta}_{t i} \theta_{t i}(\cdot) ; \quad(\cdot) \equiv\left(x_{i}, \bar{\theta}_{t}, x_{0}, \theta_{0 i} t\right)
$$

and by (E.12) and (E.5) we have

$$
\theta_{t i}=\theta_{0 i}+O(X)+O(t), \bar{\theta}_{t i}=\bar{\theta}_{0 i}+O(X)+O(t)
$$

Using (E.13 - 15) we finally obtain

$$
S^{\prime}=\frac{1}{2 t}\left(g_{\mu \nu}\left(x_{0}\right) X^{\mu} X^{\nu}+O\left(X^{3}\right)\right)-i \bar{\theta}_{1 a} \theta_{0 a}+O(X)+0(t)
$$

which we used in section 2.2, eq. (2.54)) to prove that the kernel $K_{W}^{\prime}$ has the correct limit as $t \rightarrow 0$.

It is obvious that this process can be continued to give the modified action $S^{\prime}$ to arbitrasy order in $t, X$, etc. The subtle point is the inversion necessary to express $S^{\prime}$ as a function of the proper variables. By (E.15) we can write

$$
\begin{aligned}
& \theta_{t}=\theta_{0}+X^{\mu} f_{\mu}+t g \\
& \bar{\theta}_{t}=\bar{\theta}_{0}+X^{\mu} h_{\mu}+t \ell
\end{aligned}
$$


where $f_{\mu}, g, h_{\mu}$ and $l$ are analytic functions (of $\theta_{0}, \bar{\theta}_{0}, X, t$ and the "potentials" $W$ and $B_{\mu}$ and their derivatives at $x_{0}$ ), which can be obtained by repeatedly differentiating eqs. (E.7) and the corresponding equations for $y_{k}, \varphi_{k}, \bar{\varphi}_{k}, k>$ 1. At each stage $\dot{y}_{k}(0)$ is expressed as a function of $X$, as we did in (E.10) for $\dot{y}_{0}(0)$.

Equation (E.17b) can be inverted to give $\bar{\theta}_{0}$ as a function of $\left(\bar{\theta}_{t}, \theta_{0}, x_{t}, x_{0} ; t\right)$, order by order in $X$ and $t$. For instance, to first order we have

$$
\bar{\theta}_{0}=\bar{\theta}_{1}-X^{\mu} h_{\mu}\left(X=0, \theta_{0}, \bar{\theta}_{0}=\bar{\theta}_{1}, t=0\right)-t \ell\left(X=0, \theta_{0}, \bar{\theta}_{0}=\bar{\theta}_{l}, t=0\right)
$$

This process can be continued to give $S^{\prime}\left(x_{1}, \bar{\theta}_{1}, \theta_{0}, x_{0} ; t\right)$ as a power series in $t$ (starting with $1 / t$ ) in which the coefficients are polynomials in the variables and the "potentials" and their derivatives. 


\section{REFERENCES}

1. W.A. Bardeen, Phys. Rev. 184 (1969) 1848

2. D.J. Gross and R. Jackiw, Phys. Rev. D6 (1972) 477

3. J. Wess and B. Zumino, Phys. Lett. 37B (1971) 95

4. B.Zumino, W. Yong-Shi and A. Zee, Nucl. Phys. B239 (1984) 477-507.

5. B. Zumino, Chiral anomalies and differential geometry, Lectures given at Les Houches, August 1983, Relativity, groups and topology II, p. 1291, ed. Brice S. DeWitt and R. Stora (North Holland, Amsterdam)

6. R. Stora, Algebraic structure and topological origin of anomalies, Cargèse Lectures 1983, Recent progress in gauge theories, ed. H. Lehman, NATO ASI series (New York, Plenum Press 1984)

7. W.A. Bardeen and B. Zumino, Nuel. Phys. B244 (1984) 2597-2600.

8. F. Langouche, T. Schücker and R. Stora, Phys. Lett. 145B (1984) 342

9. G. Girardi, R. Grimm and R. Stora, LAPP-TH-130 preprint.

10. E.Witten, Nucl. Phys. B.223 (1983) 422

11. O. Alvarez, I.M. Singer and B. Zumino, Commun. Math. Phys. 96 (1984) 409-417.

12. L. Alvarez-Gaumé and P. Ginsparg, Nucl. Phys. B243 (1984) 409-417.

13. L. Alvarez-Gaumé, Commun.Math.Phys.90 (1983) 161. 
14. D. Friedan and P. Windey, Nucl. Phys. B235 (1984) 395-416.

15. P. Windey, Lectures at the 23rd Cracow School of Theoretical Physics, CERN preprint TH.3578 (1983).

16. B. Zumino, Lecture at Shelter Island Conference, June 1983, LBL preprint LBL-17972.

17. T. Eguchi, P.B. Gilkey and A.J. Hanson, Phys. Reports 66 (1980) 213

18. S.S. Chern, Complex manifolds without potential theory (Springer, New York, 1979)

19. T.H.R. Skyrme, Proc. Roy. Soc. A260 (1961) 127

20. E. Getzler, Commun.Math. Phys. 92 (1983) 163.

21. J.M. Bismut, Commun.Math.Phys. 98 (1985) 213-237.

22. J.H. Van Vleck, Proc.Nat.Acad.Sci. USA 14 (1928) 178.

23. W. Pauli, Lectures on Physics Vol. 6, M.I.T. Press (1973) p. 161.

24. Ph. Choquard, Helvetica Physica Acta, Vol. 28 (1955) 89-157.

25. H. Goldstein, "Classical Mechanics", Addison-Wesley (1980).

26. R. Arnowitt, P. Nath and B. Zumino, Phys. Lett. 56 (1975) 81.

27. C. Itzykson and J.B. Zubert, "Quantum Field Theory", Mc Graw-Hill (1980).

28. L.D. Faddeev, A.A. Slavnov, "Gauge Fields: Introduction to Quantum Theory", Benjamin/Cummings(1980). 
29. B.S. DeWit, Phys. Rep. (Phys. Lett. C) 19, No. 6 (1975) 295-357.

30. R. Casalbuoni, Nuovo Cimento 33A, No. 3 (1976) 389.

31. A. Barducci, R. Casalbuoni and L. Lusanna, Nucl. Phys. B124(1977) 93-108.

32. A. Barducei, R. Casalbuoni and L. Lusanna, Nucl. Phys. B124(1977)521538.

33. M. S. Marinov, Phys. Reports, 60, No.1 (1980) 1-57.

34. F. A. Berezin and M. S. Marinov, Annals of Physics 104 (1977) 336-362. 
This report was done with support from the Department of Energy. Any conclusions or opinions expressed in this report represent eolely those of the author(s) and not necessurily those of The Regents of the University of California, the Luwrence Berkeky Laboralory or the Department of Enercy.

Reference to a company or product name does not imply approval or recommendation of the praduct by the University of California or the U.S. Department of Energy to the exclusion of others that may be suitable. 